Article

\title{
Analysis of a Signalized Intersection with Dynamic Use of the Left-Turn Lane for Opposite through Traffic
}

\author{
Yongtao Zheng, Xuedong Hua, Wei Wang *, Jialiang Xiao and Dongya Li \\ Jiangsu Key Laboratory of Urban ITS, Jiangsu Province Collaborative Innovation Center of Modern Urban \\ Traffic Technologies, School of Transportation, Southeast University, Nanjing 211189, China; \\ zyt_seu@163.com (Y.Z.); qdurgk@163.com (X.H.); xiaobenny@126.com (J.X.); lidongya@seu.edu.cn (D.L.) \\ * Correspondence: wangwei_transtar@163.com
}

Received: 15 June 2020; Accepted: 10 September 2020; Published: 12 September 2020

\begin{abstract}
For signalized intersections with a quantitative imbalance between the approach and exit lanes, this study proposes a method for the dynamic use of the left-turn lane for opposite through traffic to improve the efficiency of the signalized intersection. The complete scheme of this method mainly consists of three parts: the location routes of the dynamic lane, the temporal scheme, and the spatial scheme. To demonstrate the effectiveness of this method, a simulation scheme comparing the intersection with and without the dynamic lane is designed using VISSIM. The parameters of the simulation model, including the traffic simulation environment parameters and built-in VISSIM models, are calibrated by the field data collected from an intersection in Nanjing, P. R. China. The results of the simulation indicate that this method decreases the average delay and average queue length of the intersection effectively for the intersection with a quantitative imbalance between the approach and exit lanes under the conditions of different through volumes or left-turn ratios. The maxima were $46.9 \%$ (average delay) and $68.3 \%$ (average queue length) for all situations. Lastly, some suggestions are presented for the application of this method.
\end{abstract}

Keywords: signalized intersection; efficiency optimization; dynamic lane; VISSIM

\section{Introduction}

Signalized intersection approaches are usually channelized to serve directional flow and facilitate signal control [1]. More than one approach lane is used to serve one single directional flow due to large traffic demands in some cases, and the exact number of such lanes is easy to calculate according to the volume. Until now, however, there was no definitive method to determine the number of exit lanes. For engineering practice, experience demonstrates that it is more acceptable if the exit lane amount is greater than the approach amount in an independent phase time to ensure that the traffic flows smoothly and steadily. Moreover, studying the adjustment factors of the saturation flow rates at signalized intersections can also help determine the layout of the intersection, including the exact number of lanes in each direction [2].

However, a special kind of intersection still exists in the downtown areas of cities in developing countries like China, whose number of exit lanes is less than that of the approach lanes. This special kind of intersection features a quantitative imbalance between the approach and exit lanes-i.e., an imbalanced intersection. Figure 1 shows a typical imbalanced intersection located in the central business district of Nanjing, P.R. China. The intersection is crossed by Hongwubei Road (N-S) and Zhongshandong Road (E-W), both of which are main streets with large volumes. There are three through lanes in the southern approach but only two lanes in the northern exit. Due to the decrease in lanes, a merging conflict originating from the through flow is generated in the intersection area alongside the existing conflicts. For through vehicles, drivers have to decrease their speed to avoid 
crashes. With a large traffic volume, this kind of behavior leads to a queue in the intersection area and sometimes spillover. As Figure 1 illustrates, when the green phase is over (the amber of the through phase is circled in red), there are still queues of through vehicles remaining in the middle of the intersection because of this conflict. Additionally, this queue prevents the vehicles from passing through the intersection smoothly in the next phase. Hence, a decrease in lanes has negative influences not only on through vehicles but also on the entire intersection.

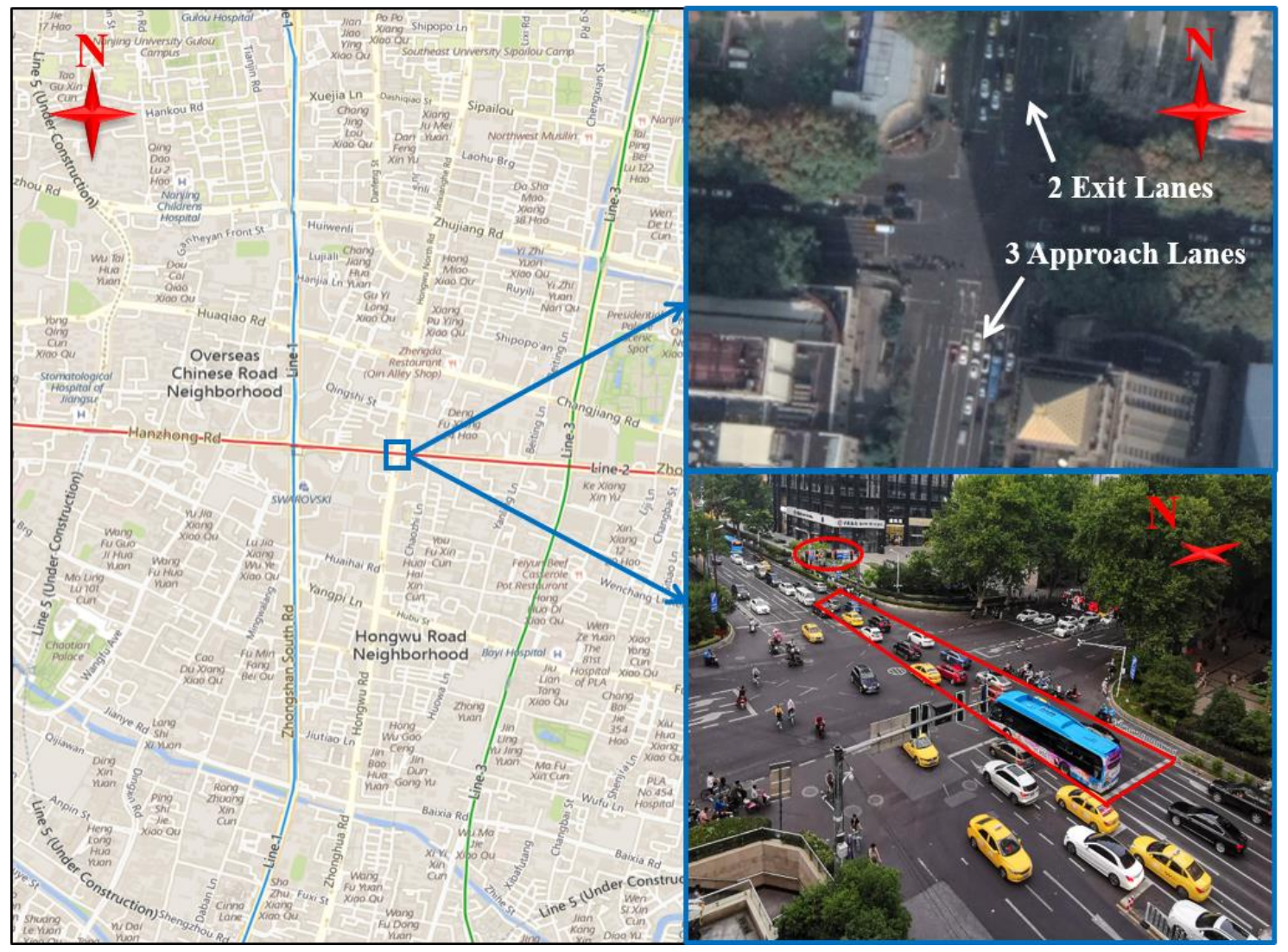

Figure 1. A typically imbalanced intersection.

To optimize an imbalanced intersection, different kinds of solutions have been proposed in recent years. The first kind involves adopting unconventional intersection geometric designs, such as a median U-turn design, bowtie, or superstreet [3-5]. The second kind of solution is a continuous flow intersection [6-9], in which an additional pre-signal is used to resolve the conflict between the left-turn and opposing through vehicles. The third kind of solution is a tandem intersection $[10,11]$, in which the pre-signal is used to reorganize the upstream traffic flow to realize cooperative control; upstream signalized crossover is one of its typical applications [12]. The performance of the solutions above has also been evaluated with respect to travel time, delays, queue length, and safety [13-17]. A more probable solution to an imbalanced intersection is the addition of an auxiliary through lane at the exit. Previous studies focused on the length and utilization of the auxiliary through lane. Yan [18] proposed a new unconventional design by allocating exit lanes appropriately based on movement, thereby creating opportunities for capacity improvement. Guell [19] indicated the relationship between intersection capacity and the length of the auxiliary through lane and presented theoretical considerations for determining the length. Tarawneh [20] outlined the utilization trends of auxiliary through lanes influenced by length and other factors. Lee [21] noted that the correlation between traffic density and the utilization of auxiliary through lanes is positive.

Even though the effectiveness of these methods has been demonstrated in both theory and practice, their application is limited by some other conditions. For example, an auxiliary through lane can be 
restricted by the use of land. In Figure 2a, the imbalanced intersection is the same as the intersection shown in Figure 1. The construction boundary of the intersection has been determined for decades. The determination of the boundary is performed under the background of a low traffic volume, and after decades of development, the surrounding constructs and facilities have been present for a long time, such as the underpasses shown in Figure $2 b$. Thus, when the traffic volume is large at present, there is not enough space to expand an auxiliary through lane. However, if some constructs and facilities are dismantled, the space used for an auxiliary through lane can be created. However, behavior like this will entail large costs and have a negative influence. Hence, the method of using an auxiliary through lane is unsuitable for this kind of imbalanced intersection.

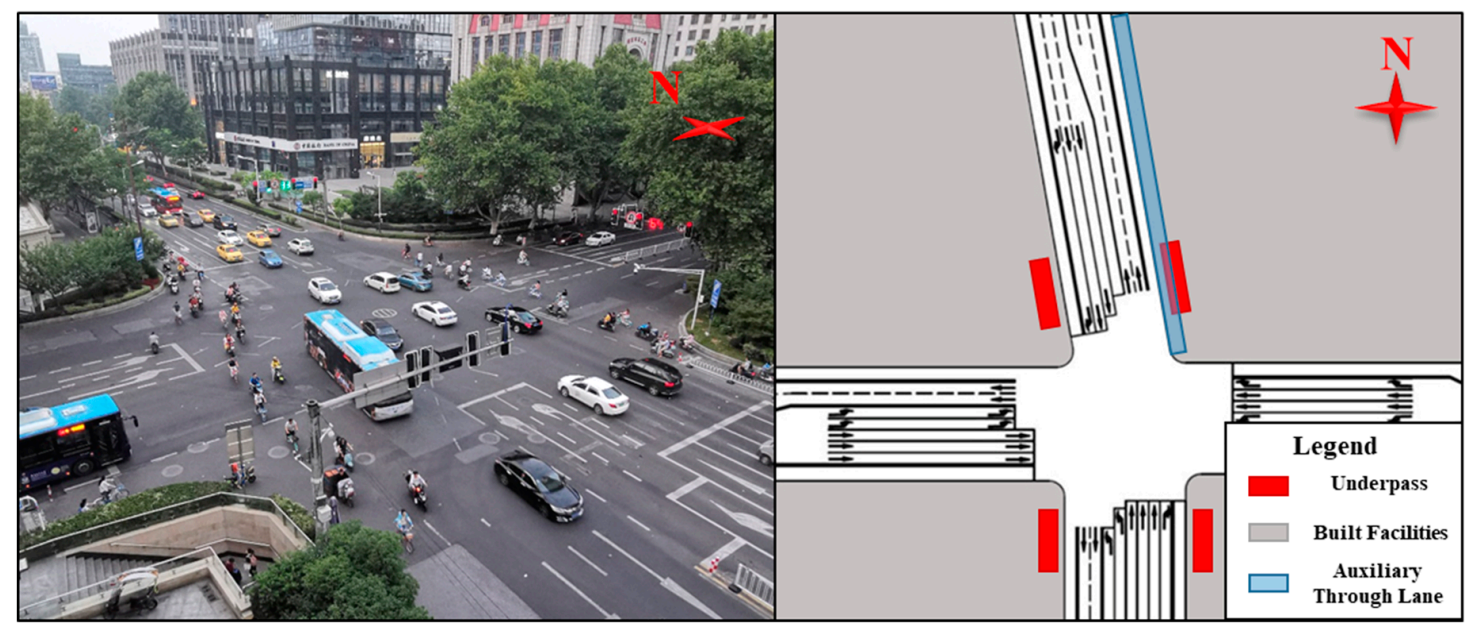

(a)

(b)

Figure 2. (a) The scene of the imbalanced intersection. (b) The restricted use of land for an imbalanced intersection.

Recently, the concept of the dynamic use of an exit lane for left turns was presented [22,23]. This method permits the left-turn vehicles to occupy the opposite exit lane during a specific time to increase the left-turn capacity of the intersection; the relevant impact and optimization methods of operation have also been evaluated [24]. Zhao J. presented an unconventional intersection design using exit lanes for left-turn (EFL) intersections. Basing on a saturation flow rate adjustment model for EFL control, the researchers considered five influencing factors and calibrated them via field data [25]. Zhao Y. proposed a capacity estimation model for an EFL intersection based on research on the geometric design and analytical procedures for estimating delays [26,27]. Chen S. built an optimization model for an EFL intersection to improve signal timing and traffic flow operations [28]. Chen Q. developed a cellular automaton model for EFL signalized intersections and verified the model via field data [29]. Liu proposed a modified shockwave-based method for estimating the maximum left-turn queue length for an EFL intersection and proved that the red time duration and offset between the main and pre-signal greatly affect the queue length [30]. Wu then proposed an actuated signal control strategy for an EFL intersection by introducing an extra phase between the pre-signal and the main signal. An optimization procedure was also proposed for optimizing the contraflow left-turn design based on the analysis model [31,32]. Zhao J. and Guo evaluated the safety of and driver responses to EFL intersections by studying the behavior of left-turn maneuvers using field data collected in China [33-35].

Inspired by the design of EFL, a method for the dynamic use of the left-turn lane for opposite through traffic (LFT) is presented in this paper. Figure 3 shows the design of this method, which permits the opposite through vehicles to occupy the dynamic lane (which is also used for left-turns) temporarily during the through phase time; meanwhile, the left-turn vehicles controlled by the pre-signal wait at the pre-stop line. The common points of EFL and LFT are as follows: (1) the dynamic use of the lane. In both EFL and LFT, in the through phase in the relevant direction, the lane is used as an exit lane for 
the through vehicles to leave the intersection, and in the left-turn phase of this direction, the lane is used as an approach lane for left-turning vehicles to enter the intersection. (2) Pre-signal. Both EFL and LFT need a pre-signal to tell the left-turn vehicles when they can drive into the dynamic lane, and the phase of the pre-signal is connected with the main signal. (3) Full use of time and space resources. The most notable advantage of EFL and LFT is their utilization of idle lane resources in a signal cycle to improve the ability and operating efficiency of the intersection. In EFL, during the through phase of the intersecting direction, the exit lane in this direction is idle. At this time, left-turning vehicles are allowed to enter the dynamic lane, yielding many left-turning vehicles passing in a cycle. In LFT, by controlling the left-turning vehicle at the pre-stop line, an additional exit lane is added for the straight vehicles in this direction, and the number of straight vehicles passing through is increased.

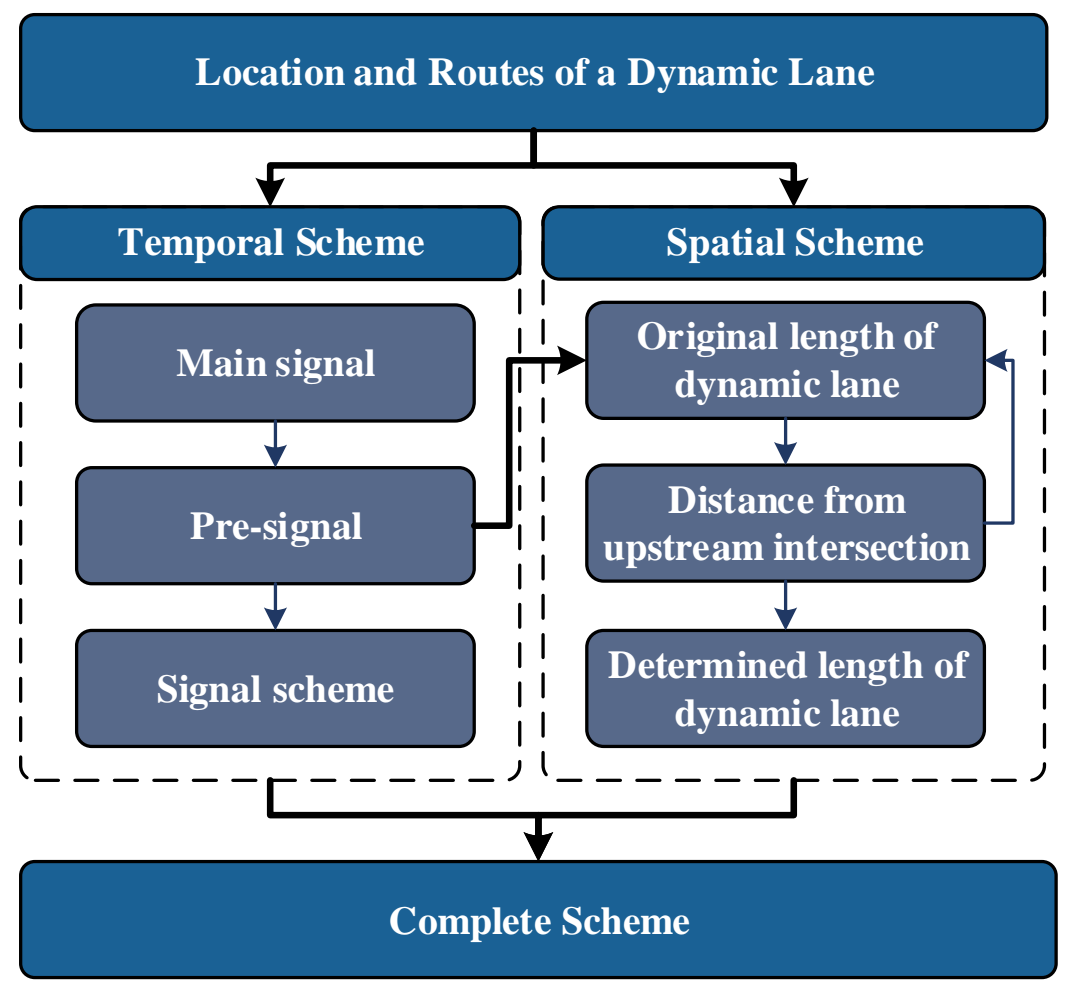

Figure 3. Structure of the methodology.

However, there are also some differences between EFL and LFT. (1) The location of the dynamic lane. In EFL, one or two exit lanes are used as dynamic lanes, but in LFT, the left-turn lane in the approach is used as the dynamic lane. (2) Optimization object. EFL is optimized for left-turning vehicles. By dynamically using exit lanes, the number of the left-turn lanes is increased, and the ability of left-turning vehicles to pass through the intersection is improved without changing the phase timing. LFT is optimized for straight vehicles. By dynamically using the left-turn lane, the number of exit lanes is increased, and the operational efficiency of the intersection is improved. (3) Application object. EFL can be applied to conventional intersections, but LFT is only applicable to imbalanced intersections, where the number of exit lanes is less than the number of approach lanes.

This article proposes a set of methods for LFT. This method consists of two significant factors: the dynamic lane and the signal phase of the intersection with respect to space and time. The application of this method solves the quantitative imbalance between the approach and exit lanes of the signalized intersection by using timing and channelized adjustments instead of expanding to another lane. The present study addresses the following research questions: (1) What conditions should be satisfied to apply this method? (2) How can we determine the length of the dynamic lane while considering safety? (3) How much is the efficiency of the intersection improved by using this method? The results 
of this study potentially provide a new perspective for engineers and designers to solve the difficulties of special intersections.

The remainder of the paper is structured as follows. First, the methodology for the dynamic use of the left-turn lane for opposite through traffic from the perspectives of time and space is illustrated in the second section. The third section describes the data collection, simulation model calibration process, and the design of the simulation scheme. The simulation results for a typical imbalanced intersection using (or not using) the dynamic lane, with a comparison and analysis, are presented in the fourth section. The fifth section discusses the performance of this method in another type of channelized intersection, the critical condition of this method, and the potential safety risks introduced by this method. Conclusions and suggestions for the dynamic use of the left-turn lane for opposite through traffic are given in the last section.

\section{Methodology}

Figure 3 illustrates the structure of the methodology in this section. First, the location and vehicle routes using dynamic lanes are outlined. Then, the scheme of the dynamic lane for application is explained and divided into temporal and spatial schemes. The temporal scheme solves the problem of signal control, while the spatial scheme determines the length of the dynamic lane with consideration of the two constraint conditions.

\subsection{Location and Routes of a Dynamic Lane}

A dynamic lane is a channelized lane serving different directional vehicles at different times. The lane is located in the middle of the approach lane and exit lane, as shown in the red zone in Figure 4. When a left turn is permitted, the dynamic lane serves the vehicles driving along Route 1 . When through traffic is permitted, the dynamic lane serves the vehicles driving along Route 2. Meanwhile, the left-turn vehicle waits at the pre-stop line to avoid conflict with the opposite through vehicle.

Since the exit lane is sufficient for vehicles driving along other routes, such as Route 3 and 4, the dynamic lane only serves the left-turn and through vehicles denoted by Route 1 and 2 . Therefore, a dynamic indicator is necessary for the realization of the target, which is hung on one side of the dynamic lane facing the direction that the Route 1 vehicles are coming from. The indicator shows a green down arrow when through traffic is permitted and a red cross during other times.

\subsection{Temporal Scheme}

The temporal scheme-in other words, the signal scheme of the entire intersection-consists of two significant sections: the main signal and the pre-signal. The main signal controls all directional vehicles. For simplification, an assumption is proposed:

Assumption 1: The main signal is composed of four phases, coordinated with two regulations:

(1) The left-turn phase must be followed by the through phase in one direction;

(2) The right-turn vehicle should be controlled independently.

The arguments of the first regulation are to minimize the delay of the left-turn vehicles using the dynamic lane while avoiding conflict between the two directional vehicles. If the left-turn phase follows the through phase, there are two factors of additional delays for left-turn vehicles: first, the time that the last opposite through vehicle consumes to drive away from the dynamic lane completely after the through phase has been terminated; second, the time that the first left-turn vehicle consumes to drive from the pre-stop line to the main stop line. However, the delays are averted if the left-turn phase is followed by the through phase because both incidents happen when the cross-directional phases are permitted, and there is no additional time wasted.

The arguments of the second regulation are to minimize disturbances to through vehicles from other vehicles. It is easily observed that the permitted right-turn phase generates another merge conflict between right-turn and cross-directional through vehicles. Hence, to maximize the efficiency 
of the dynamic lane, a controlled right turn is recommended. One of the feasible phase solutions is illustrated in Figure 4.

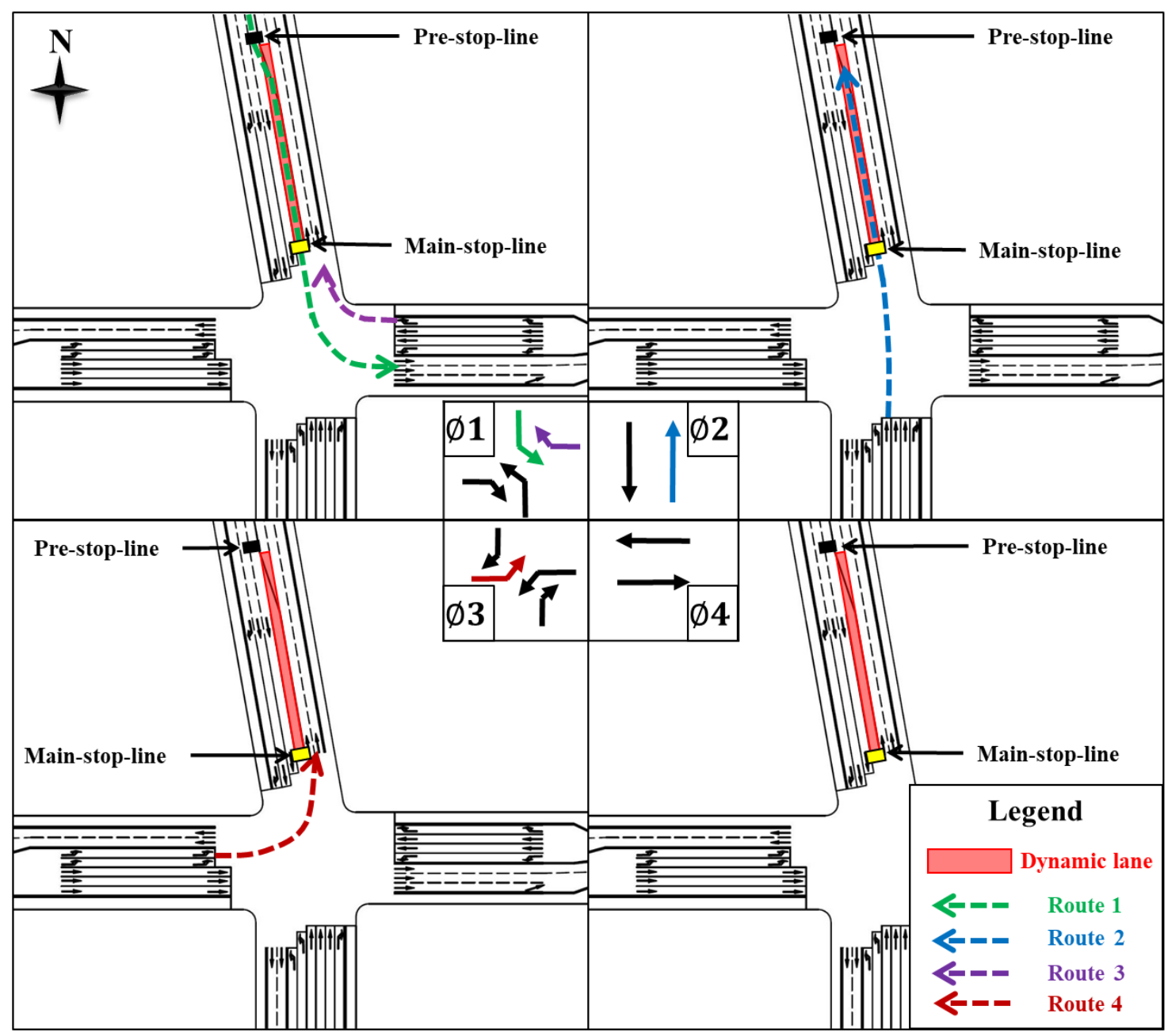

Figure 4. Relationship of time and space for a dynamic lane.

The Highway Capacity Manual 2010 [36] proposed the desirable cycle length for signal intersections as follows:

$$
\begin{gathered}
C_{d e s}=\frac{L}{1-\left[\frac{V_{c}}{s_{i}^{*} P H F(v / c)}\right]}, \\
s_{i}=s_{0} f_{w} f_{H V} f_{g} f_{p} f_{b b} f_{a} f_{L U} f_{L T} f_{R T} f_{L p b} f_{R p b},
\end{gathered}
$$

where $C_{\text {des }}$ denotes the desirable cycle length; $L$ is the total lost time per cycle; $V_{c}$ denotes the sum of the critical lane volumes; $s_{i}$ denotes the saturation flow rate of the approach; PHF denotes the peak hour factor; and $v / c$ denotes the target $\mathrm{v} / \mathrm{c}$ ratio for the critical movements in the intersection. The lost start-up time and the extension of effective green into yellow and all-red lights use the recommended value of $2 \mathrm{~s}$. Formula (2) shows the calculation approach of $s_{i}$, which is an important factor to calculate $C_{\text {des }}$. In Formula (2), $s_{0}$ denotes the base saturation flow rate; $f_{w}$ denotes the adjustment factor for the lane width; $f_{H V}$ denotes the adjustment factor for heavy vehicles in the traffic stream; $f_{g}$ denotes the adjustment factor for the approach grade; $f_{p}$ denotes the adjustment factor for the existence of a parking lane and parking activity adjacent to the lane group; $f_{b b}$ denotes the adjustment factor for the blocking effect of local buses that stop within the intersection area; $f_{a}$ denotes the adjustment factor for the area type; $f_{L U}$ denotes the adjustment factor for lane utilization; $f_{L T}$ denotes the adjustment factor 
for left-turn vehicles present in a lane group; $f_{R T}$ denotes the adjustment factor for right-turn vehicles present in a lane group; $f_{L p b}$ denotes the pedestrian adjustment factor for left-turn groups; and $f_{R p b}$ denotes the pedestrian adjustment factor for right-turn groups.

The pre-signal controls the left-turn vehicles using the dynamic lane. The green time of the pre-signal is restrained by both of the main signals and safety. However, the desirable arrangement of the pre-signal time can be outlined as follows: (1) The pre-signal time should be equal to the left-turn phase green time of the main signal; (2) it should not be necessary for the left-turn vehicles to stop again at the main signal. Due to the additional two delays for safety, to simplify the problem, another assumption is given:

Assumption 2: The sum of the cross-directional green, amber, and all-red time is sufficient to realize the desirable arrangement of the pre-signal time.

Assumption 2 requires that the traffic volume of the cross-direction must be large enough. Hence, the suitable application of this method is an imbalanced intersection crossed by two main streets with large traffic volumes. The particular arrangement of the pre-signal green time is explained in the last section of the next part.

\subsection{Spatial Scheme}

The length of the dynamic lane is restricted to two crucial factors. The first and most significant factor is safety. Vehicles driving from two opposite directions simultaneously in the dynamic lane are not permitted. Hence, to avoid this risky circumstance, one effective control strategy is to permit vehicles from one direction to occupy the dynamic lane only after all vehicles from the other direction drive away from that lane completely, which also means that the dynamic lane must be cleared and contain no vehicles. If the length is overlong, more time will be needed to clear the dynamic lane, potentially exceeding the time given by the cross-direction phases. Therefore, the limitation of the length of the dynamic lane is indispensable. Second, the independence of the intersection is another important factor. The queue formed by left-turn vehicles may spread to the upstream section due to the pre-stop line and pre-signal. If the left-turn volume is large and the length of the dynamic lane is overlong, the queue may spread into the region of the upstream intersection. However, the potential influence on the upstream intersection should be avoided by limiting the length of the dynamic lane.

In conclusion, two constraint conditions are proposed in the process of determining the length of the dynamic lane considering the two aforementioned factors:

\section{Constraint condition 1: Safety condition}

In this condition, $g_{p r e}^{m}$ denotes the maximum permitted green time of the pre-signal. $t_{0}$ denotes the time interval from the end of the through traffic green phase to the end of the left-turn traffic green phase for the researched direction. According to Figure 4, the value of $t_{0}$ equals a summary of Phase 3's green time, Phase 4's green time, Phase 1's green time, and the amber and all-red time after each of the three phases. $t_{1}$ denotes the time consumed by the left-turn car passing through the dynamic lane that drives into the dynamic lane at the end of the pre-signal green phase. $t_{2}$ denotes the time consumed by the through car driving out of the dynamic lane that passes the stop line at the end of the through green phase. $y_{\text {pre }}$ denotes the yellow time of the pre-signal. The relationship among these elements is presented as follows:

$$
g_{\text {pre }}^{m}=t_{0}-t_{1}-t_{2}-y_{\text {pre }} .
$$

The elements on the right side of the equation are calculated as follows:

$$
\begin{gathered}
t_{0}=g_{3}+g_{4}+g_{1}+3(y+a r), \\
t_{1}=\frac{L}{v_{L}}+\varepsilon_{1}, \\
t_{2}=\frac{L+w}{v_{S}}-y+\varepsilon_{2},
\end{gathered}
$$


where $g_{i}$ denotes the green time of the main signal's Phase $i(i=1,2,3,4)$, which is exhibited in Figure 4; $y$ denotes the amber time of the main signal; ar denotes the all-red time of the main signal; $L$ denotes the length of the dynamic lane; $v_{L}$ is the average speed of the left-turn vehicles passing through the pre-stop line; $w$ denotes the width of the intersection for the researched direction; $v_{S}$ is the average speed of the through vehicles passing through the stop-line; and $\varepsilon_{1}, \varepsilon_{2}$ are the artificially defined safety parameters, whose value is determined according to a real situation.

Here, $g_{p r e}^{m}$ is only decided by $L$ when the physical size of the intersection is invariable. Hence, the feasible region of $g_{p r e}^{m}$ is a crucial factor to acquire the value of $L$. When a preliminary analysis using Formulas (3)-(6) is undertaken, the negative correlation between $g_{p r e}^{m}$ and $L$ can be found. As the value of $L$ increases, the value of $g_{p r e}^{m}$ will decrease, even becoming negative if the $L$ value is too large. It is unacceptable if $g_{p r e}^{m}$ is negative. Thus, in CASE 1, the range of $L$ is established to avoid $g_{p r e}^{m} \leq 0$.

CASE 1: The range $g_{p r e}^{m} \leq 0$ is solved by Formulas (3)-(6) as

$$
L \geq \frac{v_{L} v_{S}}{v_{L}+v_{S}}\left(t_{0}-\frac{w}{v_{S}}-y_{\text {pre }}-\varepsilon_{1}-\varepsilon_{2}\right) .
$$

Here, the negative green time is inapposite. Hence, $L$ should be in the range of

$$
\left(0, \frac{v_{L} v_{S}}{v_{L}+v_{S}}\left(t_{0}-\frac{w}{v_{S}}-y_{\text {pre }}-\varepsilon_{1}-\varepsilon_{2}\right)\right) .
$$

With the restrictions of Formula (8), the dynamic lane is acceptable but not efficient enough. Supposing that the value of $g_{\text {pre }}^{m}$ is less than $g_{1}$, the amount of the left-turn vehicles passing through the intersection is decreased by the limitations of the pre-signal. This defeats the purpose of the dynamic lane. Thus, sometimes it is unnecessary to design an overly long dynamic lane. Hence, to ensure the efficiency of the left-turn vehicles, the relationship between $g_{p r e}^{m}$ and $g_{1}$ needs to be discussed. This discussion is provided for CASE 2 and CASE 3.

CASE 2: The range $0<g_{\text {pre }}^{m} \leq g_{1}$ is solved as

$$
\left(\frac{v_{L} v_{S}}{v_{L}+v_{S}}\left(t_{0}-\frac{w}{v_{S}}-y_{\text {pre }}-\varepsilon_{1}-\varepsilon_{2}-g_{1}\right), \frac{v_{L} v_{S}}{v_{L}+v_{S}}\left(t_{0}-\frac{w}{v_{S}}-y_{\text {pre }}-\varepsilon_{1}-\varepsilon_{2}\right)\right)
$$

CASE 3: The range $g_{p r e}^{m} \geq g_{1}$ is solved as

$$
\left(0, \frac{v_{L} v_{S}}{v_{L}+v_{S}}\left(t_{0}-\frac{w}{v_{S}}-y_{p r e}-\varepsilon_{1}-\varepsilon_{2}-g_{1}\right)\right] .
$$

Under these circumstances, $L$ can not only ensure the positive value of $g_{p r e}^{m}$ but also the efficiency of the left-turn vehicles. Thus, the realization of a desirable arrangement of the pre-signal is probable.

In conclusion, the length of the dynamic lane is recommended as in Formula (10). Meanwhile, the current situations of facilities should also be considered, such as the length of the channelized left-turn lane.

Constraint condition 2: Independent condition

The word "independent" means that the optimization of the researched intersection should not damage other intersections, such as the upstream intersection. In this research, this kind of damage may happen. When the dynamic lane is occupied by through vehicles, the left-turn vehicles are not permitted to drive into the dynamic lane. Thus, a queue of the arriving left-turn vehicles forms gradually. The starting point of the queue is at the pre-stop line, as shown in Figure 5. Since the pre-stop line is closer to the upstream intersection than the main stop line, the space for the queue of the left-turn vehicles is reduced by the dynamic lane. The longer the dynamic lane is, the less space there is. If the dynamic lane is too long and the traffic demands of the left-turn lane are large, the queue may spread to the upstream intersection, which may disrupt the normal operations of the 
upstream intersection. It is not recommended for the optimization of one intersection to damage another intersection. Hence, it is necessary to limit the length of the dynamic lane with consideration of the Independent condition.

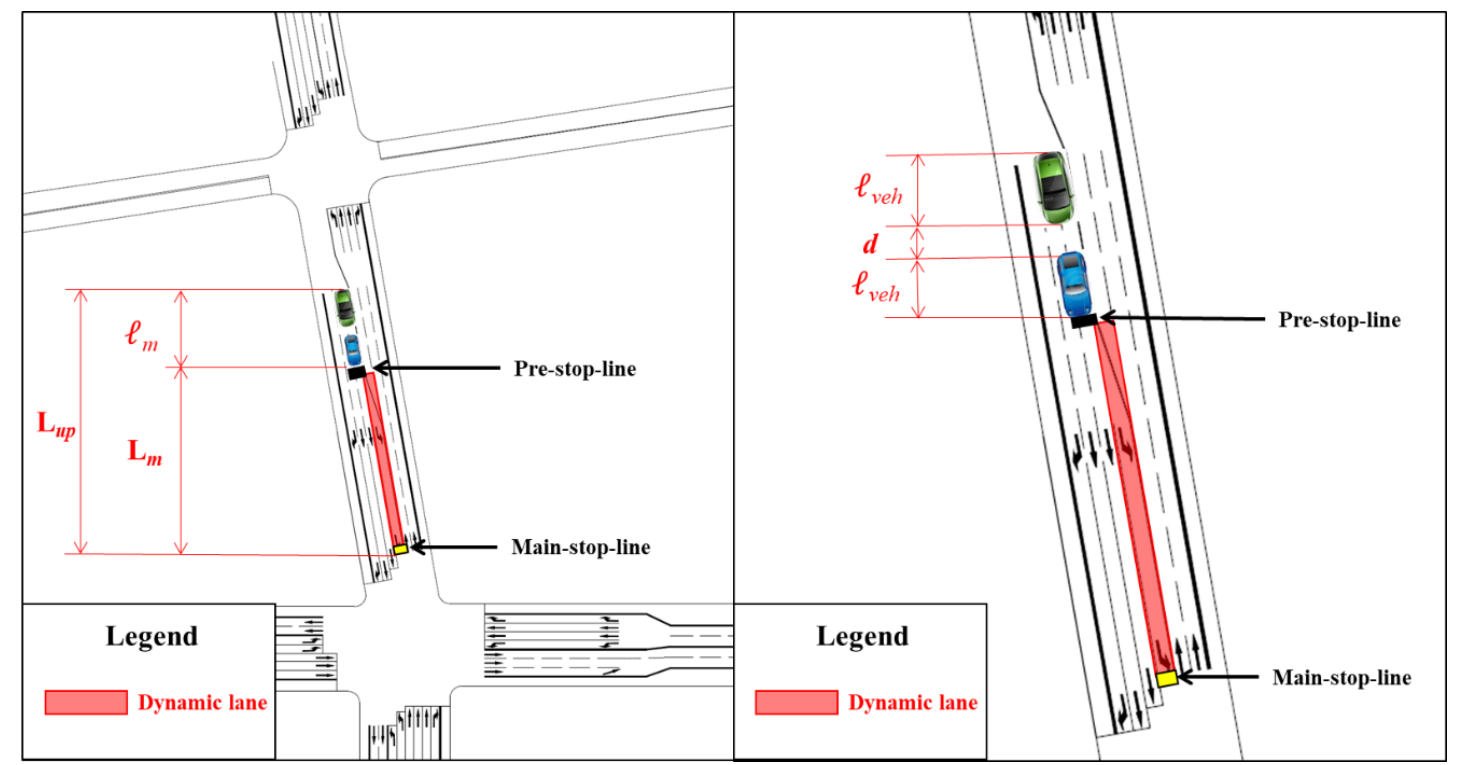

Figure 5. Relationship between the researched intersection and the upstream intersection.

Here, $L_{u p}$ denotes the distance from the stop line of the researched intersection to the start point of the channelized section of the upstream intersection; $L_{m}$ denotes the permitted maximum length of the dynamic lane with consideration of the Constraint condition $2 ; \ell_{m}$ denotes the maximum length of the queue formed by the left-turn vehicles; $N_{m}$ denotes the maximum number of vehicles in the queue; $d$ denotes the distance between two continuous vehicles in the queue; $\ell_{\text {veh }}$ denotes the average lengths of the vehicles, the recommended value of which is $5 \mathrm{~m}$. The relationship between these elements is as follows:

$$
\begin{gathered}
L_{u p}=L_{m}+\ell_{m}, \\
\ell_{m}=N_{m} \times\left(\ell_{\text {veh }}+d\right)-d .
\end{gathered}
$$

Suppose that the arrival distribution of the left-turn vehicles from the upstream intersection is a Poisson distribution [37]. The probability of reaching $n$ vehicles in $t$ time can then be described as follows:

$$
P(n)=\frac{(\lambda t)^{n} e^{-\lambda t}}{n !}
$$

where $\lambda$ denotes the vehicle arrival rate, and $t=C_{d e s}-g_{\text {pre }}$. Therefore, the constraint condition is defined as

$$
\begin{aligned}
P\left(N_{m}\right) & =\frac{(\lambda t)^{N_{m}} e^{-\lambda t}}{N_{m} !}=0, \\
N_{m} & =\frac{L_{u p}-L_{m}+d}{\ell_{\text {veh }}+d} .
\end{aligned}
$$

Formula (14) indicates that the spillover of the queue influencing the upstream intersection is not allowed.

In conclusion, considering Constraint condition 1: Safety condition and Constraint condition 2: Independent condition synthetically, the steps to determine the length of the dynamic lane are as follows:

Step 1: Determine the original length of the dynamic lane preliminarily on the basis of Constraint condition 1; 
Step 2: Check the original value $L$ on the basis of Constraint condition 2;

Step 3: If $L \leq L_{m}$ is satisfied, then the length of the dynamic lane is determined; otherwise, go back to Step 1.

With the determined value, the pre-signal green phase is designed as follows:

(1) The green time of the pre-signal is equal to the left-turn green time of the main signal, which is formulated as $g_{p r e}^{m}=g_{1}$.

(2) The pre-signal is terminated $t_{1}$ before the main left-turn phase ("terminate" indicates that the pre-signal head turns from green to amber).

(3) The beginning of the pre-signal occurs at $\left(g_{\text {pre }}+y_{\text {pre }}\right)$ before its termination.

\section{Data Collection and Simulation}

\subsection{Field Data Collection and Description}

To develop and calibrate the simulation models, field data needed to be collected. The congestion index for the top 50 cities was gathered from the AutoNavi Traffic Company [38]. The morning peak appeared from 7:00 AM to 9:00 AM, and the evening peak appeared from 5:00 PM to 7:00 PM. The 24-h congestion index in Nanjing on 10 August, 2020 is shown in Figure 6a, and the congestion index in Nanjing from 10 August to 16 August, 2020 is shown in Figure 6b. Figure 6 shows that the morning peak had the highest congestion index for the day (August 10), and the congestion index on 10 August (Monday) was also the highest for the week. Thus, it is believed that the morning peak on Monday is the most congested during the whole week in Nanjing.

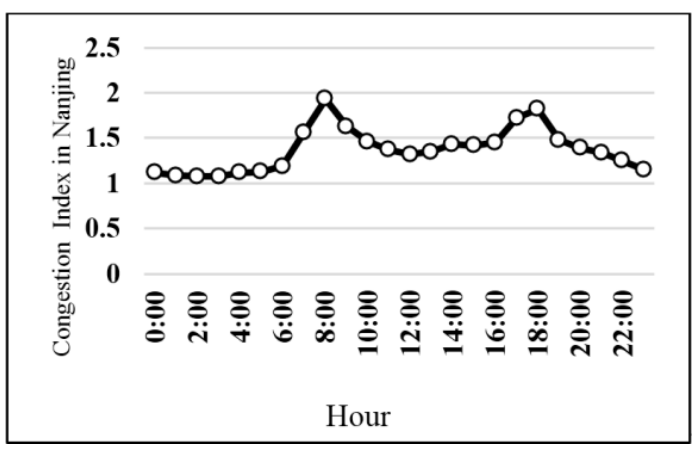

(a)

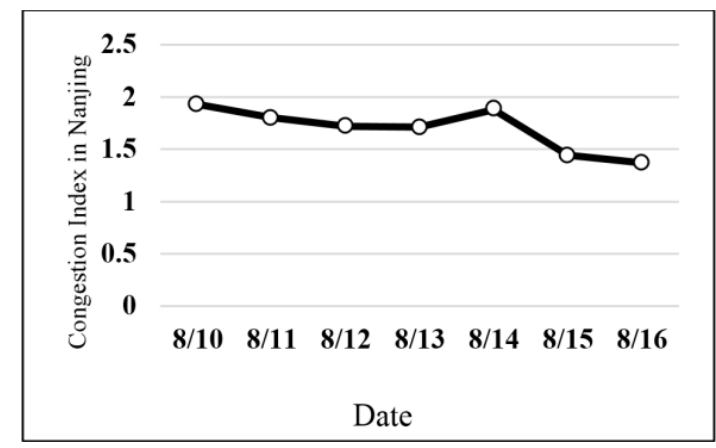

(b)

Figure 6. (a) The congestion index in Nanjing for the day. (b) The congestion index in Nanjing for the week. The real-time congestion index was gathered from the AutoNavi Company Webpage at https://report.amap.com/detail.do?city=320100.

The field data for the intersection shown in Figure 1 were also collected during both the peak and valley hours on weekdays and the weekend. The research objective was the southern approach and northern exit of Hongwubei Road. Four video cameras were set up to collect traffic data, and each of them captured one of four directions. All the cameras were placed high enough to ensure all of the vehicles could be captured clearly. Data collection was conducted from Monday, 10 August to Sunday, 16 August, 2020. For each day, the collection time was from 7:00 AM to 9:00 AM, 11:00 AM to 1:00 PM, and 5:00 PM to 7:00 PM. After a comparison, the trends of the collected data were shown to be consistent with the trends of the congestion index shown in Figure 6. Thus, we used the morning peak data on Monday to calibrate the simulation model to illustrate the most unfavorable situation. The following information was collected and used in this paper: the peak hourly flow rate (PHF Rate), transit percentage (TP), headway (used for calculation of the saturation flow rate), and travel time (TT) for each vehicle. Summary statistics of the collected data are illustrated in Table 1. 
Table 1. Summary statistics of the field data.

\begin{tabular}{cccccc}
\hline Direction & Turn & $\begin{array}{c}\text { PHF Rate } \\
\text { (pcu/h) }\end{array}$ & $\begin{array}{c}\text { Average TP } \\
\mathbf{( \% )}\end{array}$ & $\begin{array}{c}\text { Average Headway } \\
\text { (s) }\end{array}$ & $\begin{array}{c}\text { Average TT } \\
\text { (s) }\end{array}$ \\
\hline \multirow{3}{*}{$\mathrm{N}$} & $\mathrm{L}$ & 153 & & & \\
& $\mathrm{~T}$ & 672 & 4.23 & 2.43 & 61.00 \\
& $\mathrm{R}$ & 184 & & & \\
\hline \multirow{2}{*}{$\mathrm{S}$} & $\mathrm{L}$ & 211 & & 2.31 & 67.00 \\
& $\mathrm{~T}$ & 683 & 4.01 & & \\
& $\mathrm{R}$ & 174 & & 2.24 & 62.00 \\
& $\mathrm{~L}$ & 204 & & & \\
\hline \multirow{2}{*}{$\mathrm{E}$} & $\mathrm{T}$ & 738 & 5.83 & & 70.00 \\
& $\mathrm{R}$ & 133 & & 2.3 & \\
\hline
\end{tabular}

\subsection{VISSIM Model Calibration}

This research employed VISSIM, the widely used and proven microscopic traffic simulation software. To increase the accuracy of the VISSIM simulation model in the following steps, calibration was needed before the simulation and analysis. The standard calibration procedure proposed by previous studies was followed [39-43]. In this study, two types of simulation parameters had to be determined:

(1) The traffic simulation environment parameters, such as the layout of the intersection, the desired speed of different vehicle classes, traffic volumes, and transit percentages. This type of parameter can be calibrated directly using field data, as shown in Section 3.1, Field Data Collection and Description.

(2) Built-in VISSIM models, such as lane changing and travel behavior models. Several scholars have researched the calibration methods for these two kinds of models. Nima Dadashzadeh [39] proposed the complete procedures of the particle swarm algorithm (PSO) and genetic algorithm (GA) for microscopic simulation model calibration and then applied those procedures to calibrate the travel behavior models in VISSIM [40]. Chen Wang [41] applied semi-calibration to urban intersections in Shanghai, P. R. China, and selected the parameters that needed to be calibrated for the Wiedemann 74 model. In our research, the GA is used to calibrate the built-in VISSIM models, especially the Wiedemann 74 model. The process of this calibration is as follows.

To sufficiently populate the simulation model with a real intersection, according to the previous research [40], the simulation period is set to $4800(900+3600+300)$ seconds, with $900 \mathrm{~s}$ being the warm-up time at the beginning and $300 \mathrm{~s}$ being the cool-down time at the end of the simulation period. Data collection is done for just a 3600-s simulation period, excluding the warm-up periods. Since VISSIM is a stochastic model, some small differences in the results are expected with different random seeds. To address this issue, each simulation is run 10 times with different random seeds. The initial random seed is set to 40 , and the seed increment is set to 3 . For each simulation, the simulation resolution is set to 10 and runs at max speed. The reported simulation results are the average over 10 runs.

The average intersection travel times based on the field data and simulation were compared to validate the accuracy of the calibrated VISSIM model. The percentage error (PE) was used to measure the difference between the observed and simulated travel times [44]. By defining $T T_{S}$ and $T T_{R}$ as the measured travel time from simulation and reality, respectively, the PE can be obtained following Equation (16). Using the GA, the optimization process is based on a single objective function. Here, we try to minimize the error between the simulated and observed data utilizing the PE:

$$
P E=\frac{\left|T T_{S}-T T_{R}\right|}{T T_{R}} \times 100 \% .
$$


As the generation increases, the objective function value decreases. The rate of this decrease also gradually declines. This means that in the process of generational growth, the model parameters are continuously optimized. In the 34th generation, the optimal solution appears, and the objective function value is 0.76 . Thus far, the optimal combination of the three parameters has been found, which is shown in Table 2. Compared to the default values, the calibration values of the three parameters are all smaller than the default values. The calibration results show that vehicles prefer a lower safety distance, which is more dangerous. These results are consistent with the observations. Indeed, there are plenty of radical drivers at the observed intersection. These drivers prefer to stop too close to the vehicles ahead. When the signal is green, these vehicles keep a close distance to the vehicle ahead and sometimes even choose to overtake that vehicle inside the intersection, even though there are not enough chances to do so.

Table 2. Results of calibration.

\begin{tabular}{|c|c|c|c|}
\hline Parameters & Default & Calibrated & PE (\%) \\
\hline Desired safety distance between stopped cars (ax) & 2.00 & 1.13 & \\
\hline Additive of the desired safety distance (bxAdd) & 2.00 & 1.54 & 0.76 \\
\hline Multiplicative part of the desired safety distance (bxMult) & 3.00 & 2.78 & \\
\hline
\end{tabular}

\subsection{Simulation Scheme Design}

From the analysis of the dynamic lane, the elimination of merging conflicts will likely lead to an efficiency improvement. However, the dynamic lane also possibly influences the efficiency of the left-turn vehicles. Hence, to evaluate the performance of the dynamic use of the left-turn lane for opposite through traffic, a simulation scheme is designed in two parts: (1) Use of the volume of through vehicles as a variable. The southern approach ranges from 600 to $1300 \mathrm{pcu} / \mathrm{h}$ and increases by $100 \mathrm{pcu} / \mathrm{h}$ each time. Meanwhile, the northern approach ranges from 400 to $867 \mathrm{pcu} / \mathrm{h}$ and increases by $67 \mathrm{pcu} / \mathrm{h}$ because the number of through approach lanes in the north is $2 / 3$ the number in the south. The change of the flow value is used to simulate the performance of the dynamic lane under different flow situations, such as peak and off-peak hours. (2) Use of the ratio of the left-turn (represented by $\mathrm{R}$ ) vehicles as a variable. For both the southern and northern approaches, the values are $R=0.10$, 0.15 , and 0.20 . The upper limit is $1300 \mathrm{pcu} / \mathrm{h}$ and 0.2 because when the variable exceeds the upper limit, the capacity of the intersection cannot satisfy the traffic demands under the conditions of current intersection geometric designs.

In each part of the simulation, the other traffic parameters are constants, including the traffic volume and transit percentage. Moreover, the traffic volume is based on the field data and uses the adjacent integers of the original figures, such as 200 replacing 180 and 900 replacing 894 . However, the transit percentage is unchanged. For signal control, the phases of the main signal are shown in Figure 4, and the timing of the main signal and pre-signal is determined by the aforementioned methods for each simulation. Then, the length of the dynamic lane is determined as $50 \mathrm{~m}$ under consideration of the two constraint conditions, as long as the original left-turn approach lane is in the north.

The desirable cycle length of the main signal is calculated by Formula (1). The saturation flow rate of the approach is calculated by Formula (2). To simulate a real intersection, the saturation flow rate adjustment factors are determined according to reality. The value of each parameter is shown in Table 3. The settings of the simulation parameters are the same as the settings in the calibration progress, which are described in Section 3.2, VISSIM Model Calibration. 
Table 3. Value of the saturation flow rate adjustment factors.

\begin{tabular}{cccccc}
\hline Parameter & Value & Parameter & Value & Parameter & Value \\
\hline$s_{0}$ & $1900 \mathrm{pcu} / \mathrm{h} / \mathrm{ln}$ & $f_{p}$ & 1.00 & $f_{L T}$ & 0.95 \\
$f_{w}$ & 1.00 & $f_{b b}$ & 1.00 & $f_{R T}$ & 0.85 \\
$f_{H V}$ & 0.96 & $f_{a}$ & 1.00 & $f_{L p b}$ & 1.00 \\
$f_{g}$ & 1.00 & $f_{L U}$ & 1.00 & $f_{R p b}$ & 1.00 \\
\hline
\end{tabular}

Moreover, the control group and experimental group operate simultaneously. The difference between these two kinds of groups is that the northern approach of the control group is consistent with the field, but the northern approach of the experimental group is transformed by a dynamic lane. However, the parameters are identical in the two groups.

\section{Results}

\subsection{Impact of Dynamic Lane on Traffic Delay}

Delay is a significant indicator needed to evaluate the operations of an intersection and is also used to estimate a new model or method for the intersection, according to previous studies [45-48]. Yang Min used the delay as an important indicator to evaluate the influence of a modern tram under a coordinated signal priority strategy [45]. Mohan Mithum used a decrease in the delay to demonstrate the effectiveness of the proposed new method [46]. Both Liu Pan [47] and Saha Arpita [48] used the delay as the indicator to evaluate the simulation model in VISSIM. There are several similarities between this research and previous studies, such as the research object being an intersection and the delay being adopted as a crucial indicator in this research. However, it is not particularly valuable to simply explore the regularity of delay changing with independent variables because the target is to evaluate the performance of the dynamic lane compared to the field. Hence, the reduction rate of the average delay (RRAD) is selected as one of the evaluation indicators. We define $D_{E}$ as the average delay of the experimental group and $D_{C}$ as the average delay of the control group. Hence, the RRAD can be obtained by Formula (17):

$$
R R A D=-\frac{D_{E}-D_{C}}{D_{C}} \times 100 \% .
$$

Figure 7a,b illustrate the performance of the dynamic lane for the RRAD of the entire intersection and through vehicles. In Figure $7 \mathrm{a}$, when the ratio of left-turn vehicles is fixed, the influence of the dynamic lane on the RRAD of the entire intersection increases with the volume of through traffic. Under a low through volume, especially when the value is less than $800 \mathrm{pcu} / \mathrm{h}$, the performance of the dynamic lane is not sufficient. When the volume increases continually, the value of the RRAD is more than $10 \%$, which shows that the contribution of dynamic lanes is important. It can be observed that the dynamic lane eliminates merging conflicts such that the average speed of the through vehicles is improved. When the green time is consistent, more vehicles can pass through the intersection at a higher speed. Particularly, when the through volume is more than $1200 \mathrm{pcu} / \mathrm{h}$, there is spillover in the control groups, but the experimental group operates smoothly as usual, and the RRAD becomes larger under this circumstance. The maximum of the RRAD can reach $46.9 \%$. When the volume is fixed, the influence of the dynamic lane on the RRAD of the entire intersection increases with an increase in the left-turn ratio in most situations. Thus, when the ratio increases, the total volume passing through the intersection increases, magnifying the performance of the dynamic lane.

Figure $7 \mathrm{~b}$ illustrates the tendency of the RRAD of through vehicles, which is consistent with the RRAD of the entire intersection. Therefore, it is assumed that a decrease in through vehicle delays mainly contributes to a decrease in the entire intersection's delays due to the dynamic lane. In other words, the dynamic lane mostly influences the through vehicles. 


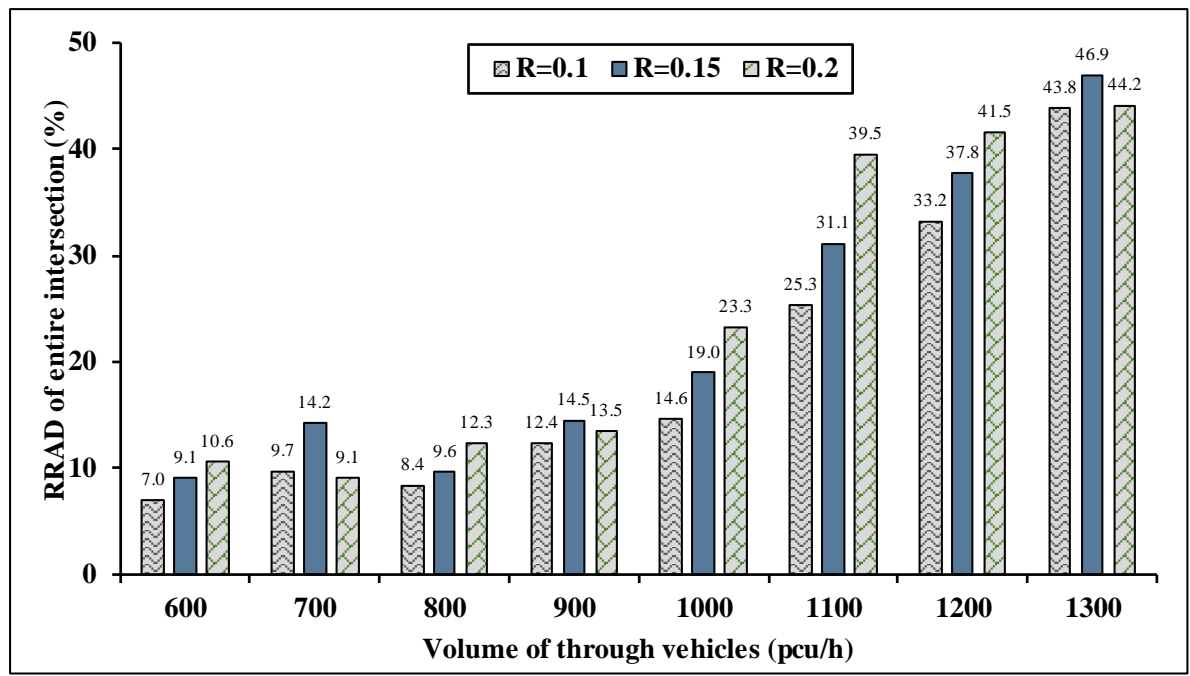

(a)

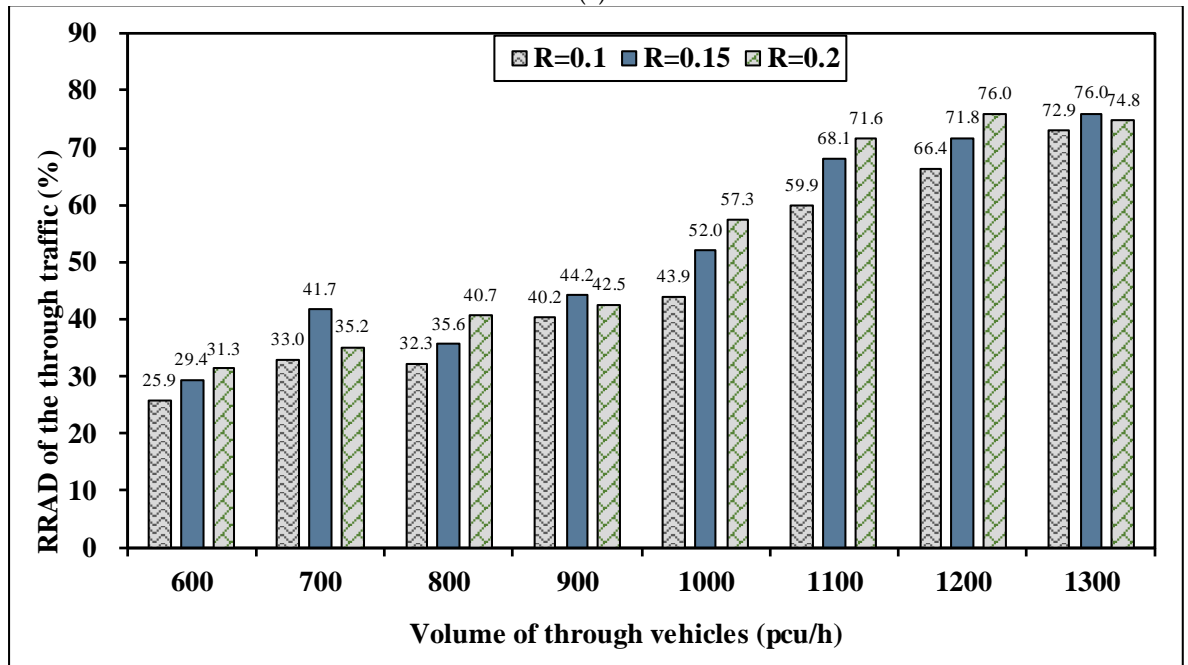

(b)

Figure 7. Impact on traffic delay: (a) reduction rate of the average delay (RRAD) of the entire intersection; (b) RRAD of the through traffic.

\subsection{Impact of Dynamic Lanes on Queue Length}

Queue length is another significant indicator to evaluate the operation of an intersection, according to previous studies. Zhang Han [49] indicated that queue length is a crucial measure of intersection performance. Liu Shiwei [50] used the queue length as an important indicator to estimate a signal timing optimization algorithm for an intersection connected to an urban expressway. Based on previous research, in this study, the queue length is used as another important indicator for evaluating the operational efficiency of the intersection, except for the delays. Like the definition of the RRAD, it makes little sense to simply consider changes in the absolute value of the queuing length at an intersection as this research focuses on how the dynamic lane affects the efficiency of intersection operations. Hence, the comparison between the intersection with the dynamic lane (experimental group) and the intersection without the dynamic lane (control group) adopts the reduction rate of the average queue length (RRAQ) to evaluate the performance of the dynamic lane. The calculation method of the RRAQ is provided in Formula (18). In this formula, $Q_{E}$ represents the average queue length of the experimental group, and $Q_{C}$ represents the average queue length of the control group.

$$
R R A Q=-\frac{Q_{E}-Q_{C}}{Q_{C}} \times 100 \%
$$


Figure 8a shows the RRAQ of the entire intersection. From the perspective of the absolute value, the effect of the dynamic lane on reducing the queue length of the entire intersection is significant. Regardless of whether the volume of through vehicles is large or small, the overall queuing length at the intersection is reduced by more than $10 \%$. When the volume is $1300 \mathrm{pcu} / \mathrm{h}$ and the left-turn ratio is 0.2 , the RRAQ reaches the highest value of $68.3 \%$. From the perspective of change trends, the trend of the RRAQ along with the volume of the through vehicles and the left-turn ratio is the same as that of the RRAD. Since the dynamic lane eliminates the merging conflict point, the secondary queuing caused by the merging of the through vehicles inside the intersection is also eliminated. With an increase in the volume of the through vehicles, when there is no dynamic lane, the queue formed by the merging conflict will become increasingly longer and possible produce a spillover. When setting the dynamic lane, there will be no secondary queuing. Therefore, the RRAQ will increase with an increase in the volume of the through vehicles. When the volume is fixed, the influence of the dynamic lane on the RRAQ of the entire intersection increases with an increase in the left-turn ratio in most situations. Like with the tendency of the RRAD, when the ratio increases, the total volume passing through the intersection increases as well, so the performance of the dynamic lane is also magnified.
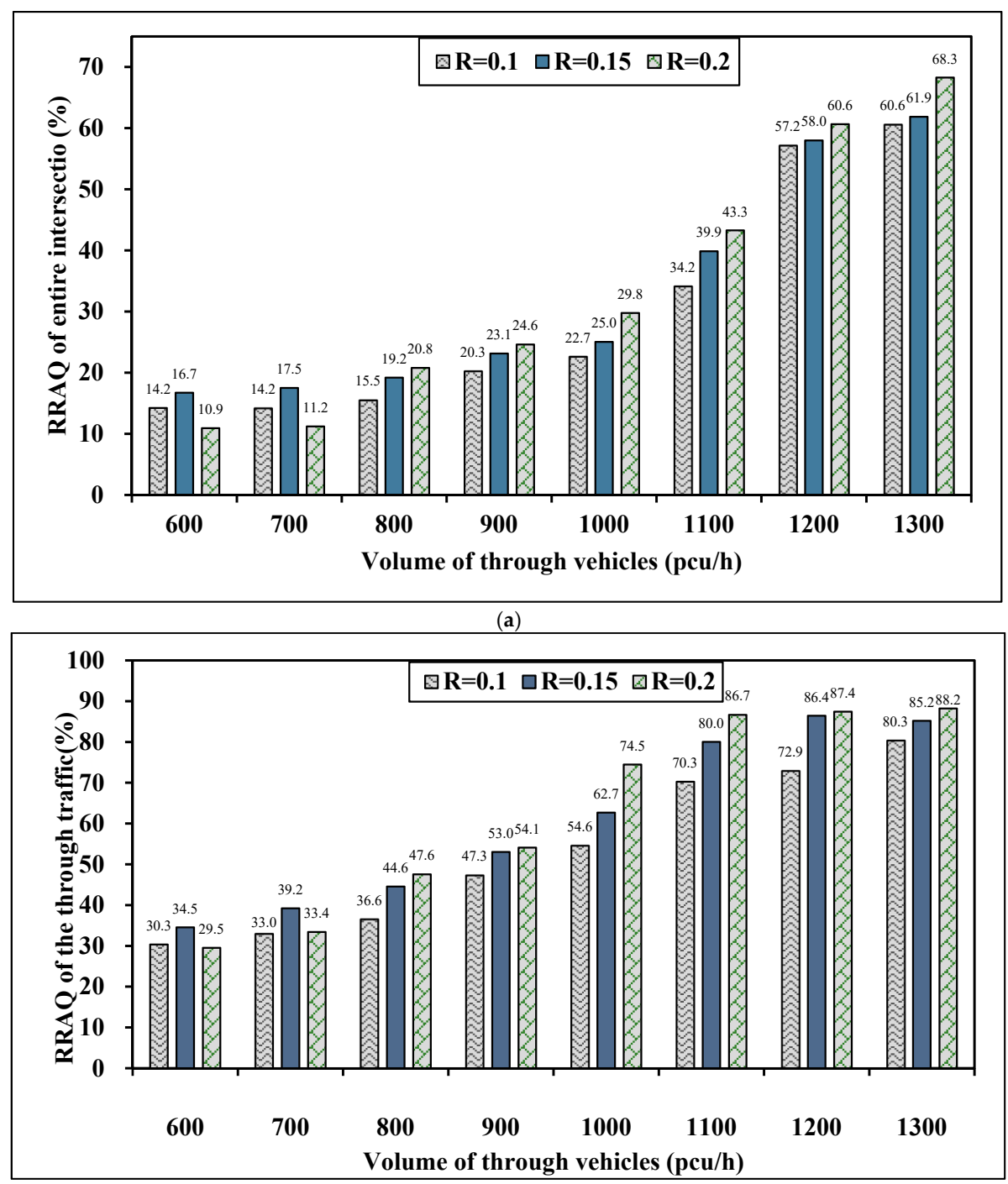

(b)

Figure 8. Impact on queue length: (a) reduction rate of the average queue length (RRAQ) of the entire intersection; (b) RRAQ of the through traffic. 
As Figure $8 \mathrm{~b}$ shows, the RRAQ of the through vehicles presents a similar variation law. Comparing these two figures, it can be observed that the value in Figure $7 \mathrm{~b}$ is larger than that in Figure 7a under the same conditions, so a decrease in the entire intersection queue length is mainly due to a decrease in the through queue length.

\section{Performance of the Dynamic Lane for Another Type of Channelized Intersection}

\subsection{Setting Method for the Dynamic Lane}

The dynamic lane can decrease the average delay significantly for this kind of the intersection in Figure 1. However, there are various forms of channelization at intersections, especially for the left-turn lane. Generally speaking, there are two forms of widening: widening to the inside and not widening to the inside. We previously discussed the operation of dynamic lanes at intersections where the left-turn lanes widen to the inside. Hence, the performance of the dynamic lane still needs to be evaluated for when the left-turn lane does not widen to the inside at the intersection. Figure 9a shows another type of imbalanced intersection. The number of the northern exit lanes is still less than that of the southern approach lanes. However, the left-turn lane of the northern approach does not widen, which is different from the intersection shown in Figure 1.

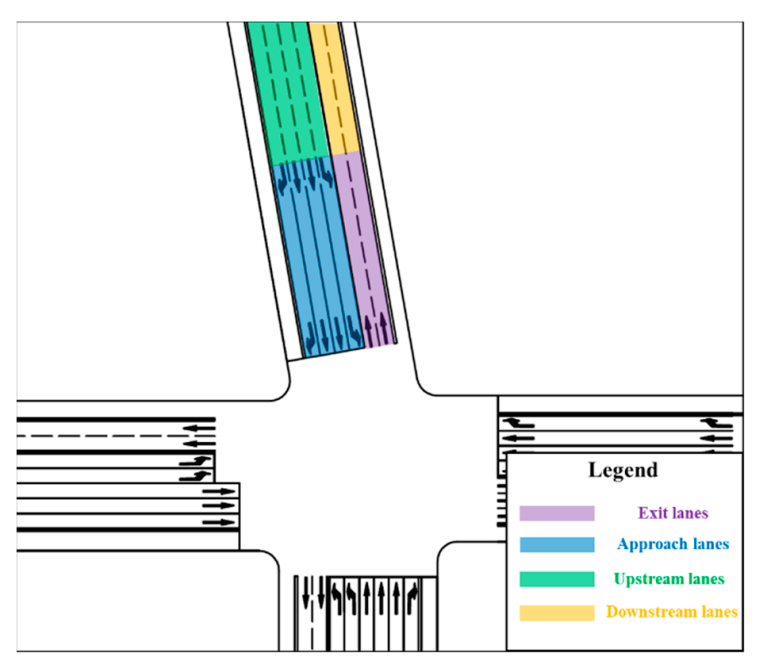

(a)

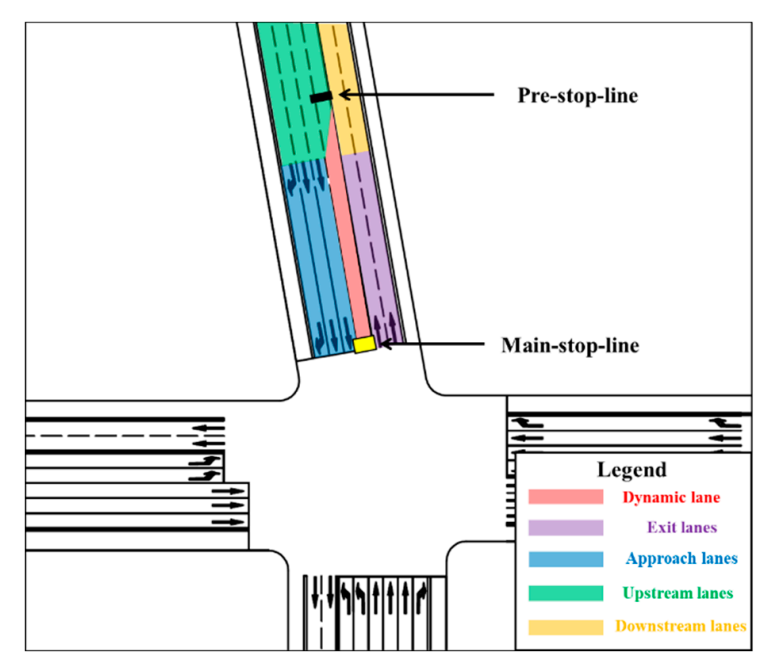

(b)

Figure 9. (a) Another type of channelized intersection; (b) the layout of the dynamic lane.

In this case, dynamic lanes can also be used. The setting method for such lanes is the same as that shown above. In terms of space, the original left-turn lane is changed into a dynamic lane, which can be used for both left-turning vehicles and the opposite through vehicles. In terms of signals, the phase setting and timing methods can also follow the methods shown in Section 2.1, Location and Routes of the Dynamic Lane and Section 2.2, Temporal Scheme. For the length of the dynamic lane, the progress of determination can follow the method in Section 2.3, Spatial Scheme. Comparing Figures 4 and 9, an important difference is found between the dynamic lanes in these two situations. In Figure 4, due to the existence of dynamic lanes, the merging conflict point is eliminated. In Figure 9, even if the dynamic lane is selected to balance the quantitative difference between the approach and exit, the merge conflict will not be eliminated but simply moved to the downstream section of the exit.

To evaluate whether the dynamic lane is effective, another comparative simulation is developed. This simulation scheme is based on the proposed scheme, but the layout of the lanes is slightly different in the north of the intersection: (1) Here, there are four channelized lanes on the approach, but the number of upstream lanes on the approach is still four; (2) two lanes are included at the exit, but the number of downstream lanes at the exit is also two, as Figure 9a shows. The sets of variables and parameters in this simulation scheme are identical to those of the proposed simulation scheme, which is 
stated in Section 3.3, Simulation Scheme Design. The same evaluation indicators are used to evaluate the performance of the dynamic lane.

\subsection{Simulation Result and Analysis}

We use the same indicators to evaluate the performance of the dynamic lane in this case, such as the RRAD and RRAQ. The results of the simulation are shown in Figures 10 and 11. Figure 10 shows the trend of the RRAD, as the flow of straight vehicles increases under different left-turn ratios. On the whole, as the volume of through vehicles increases, the RRAD value increases, and, as the ratio of left-turn vehicles increases, the RRAD value also increases.

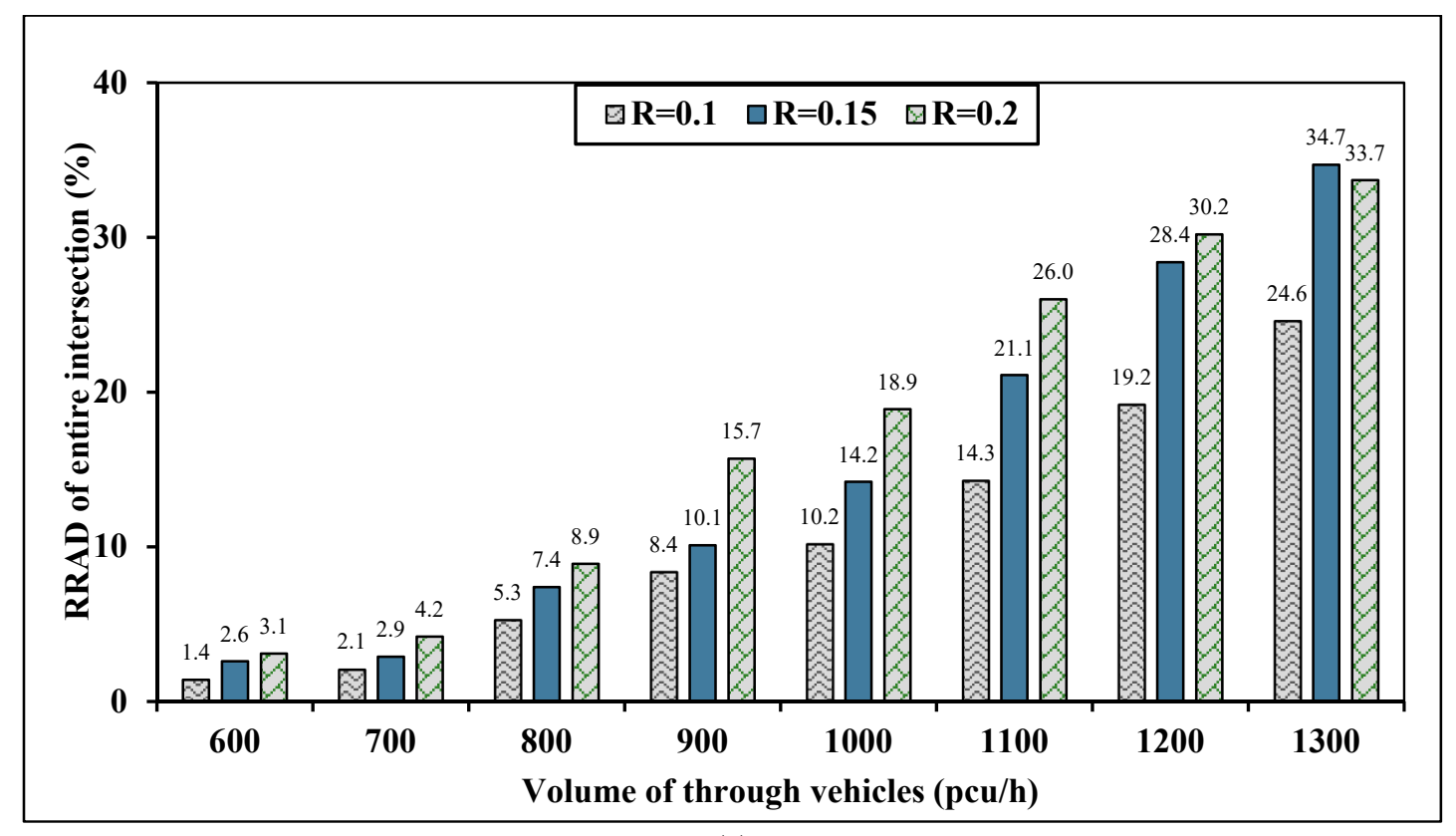

(a)

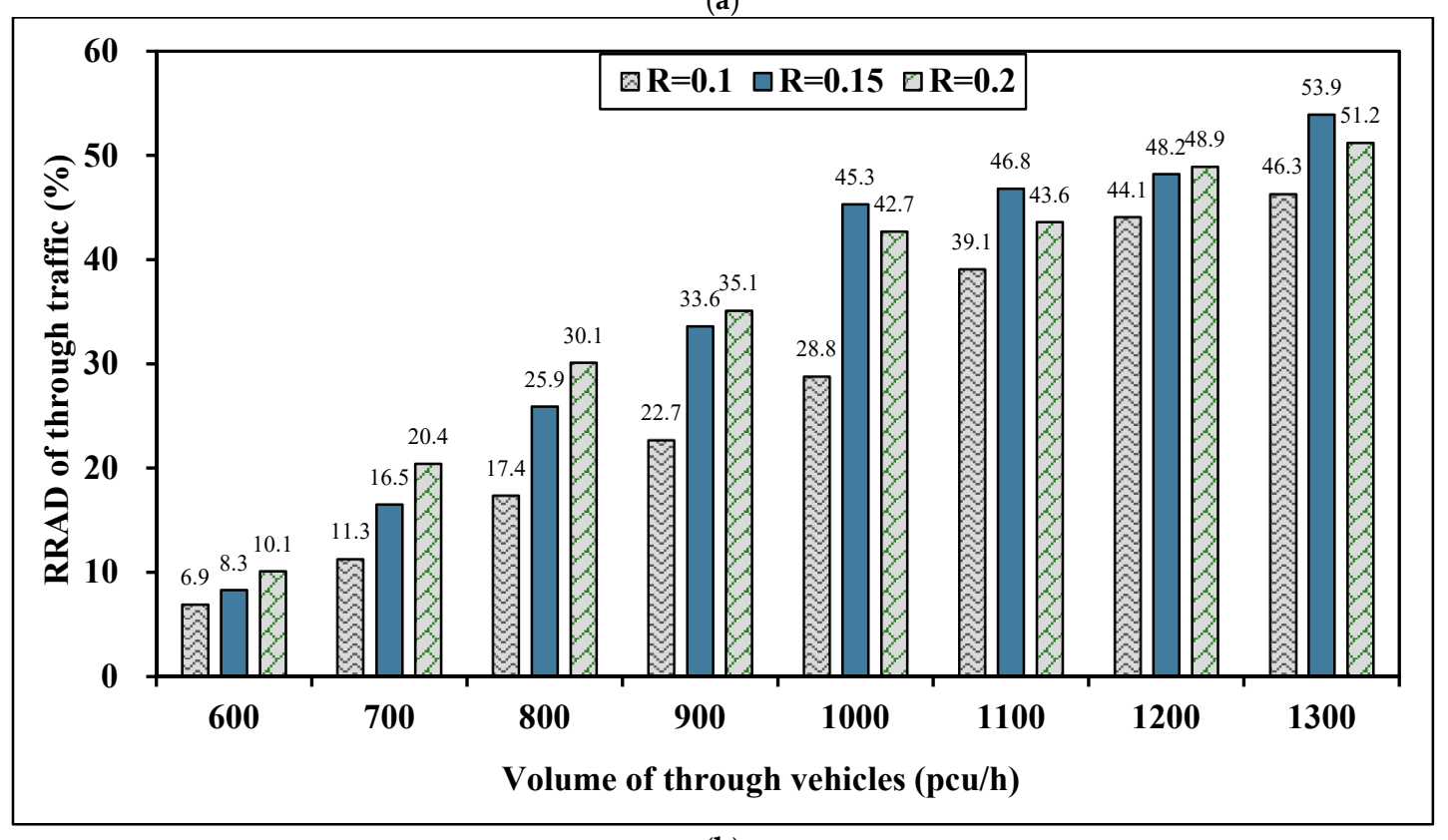

(b)

Figure 10. Impact on traffic delay: (a) RRAD of the entire intersection; (b) RRAD of the through traffic. 


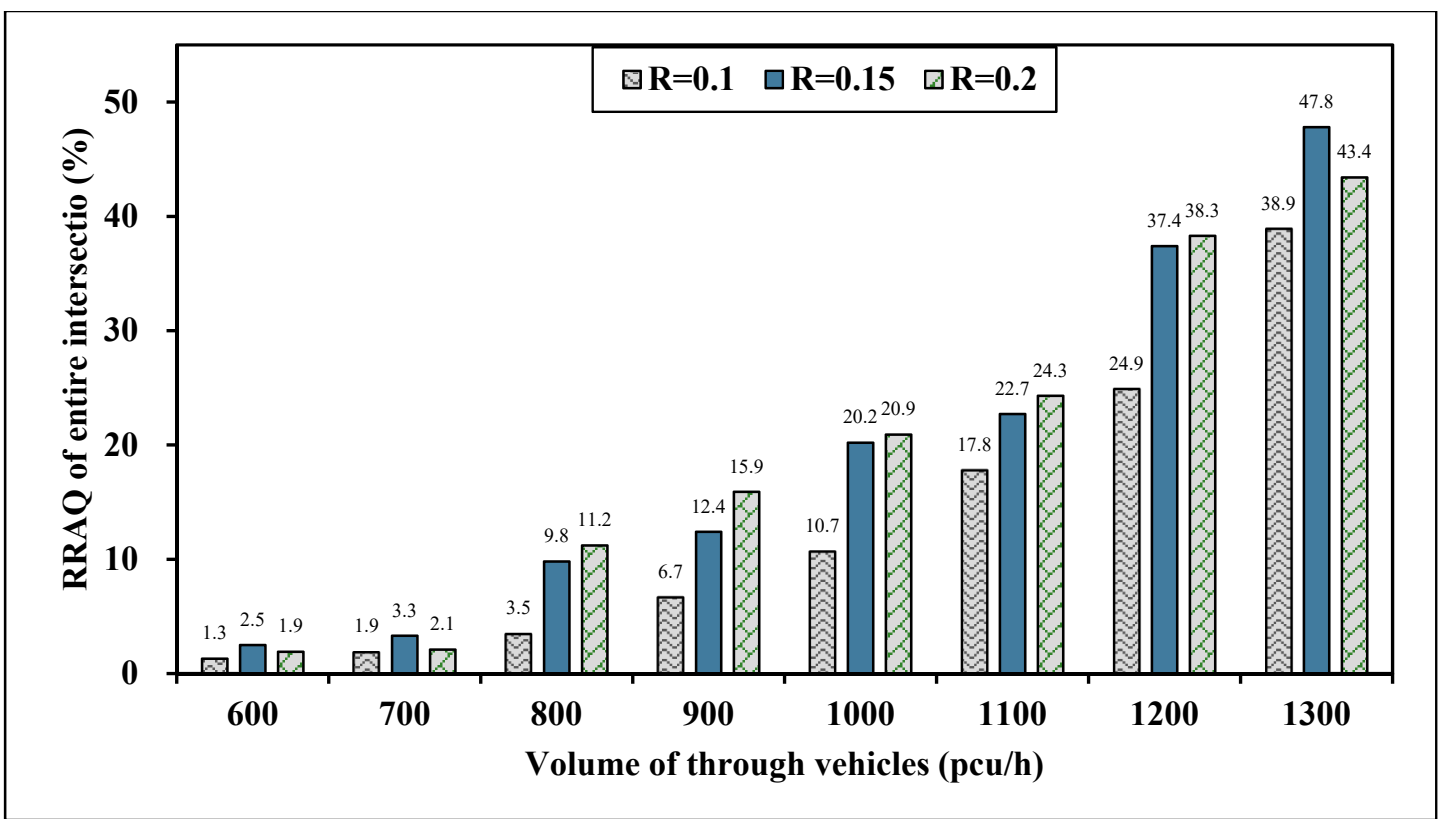

(a)

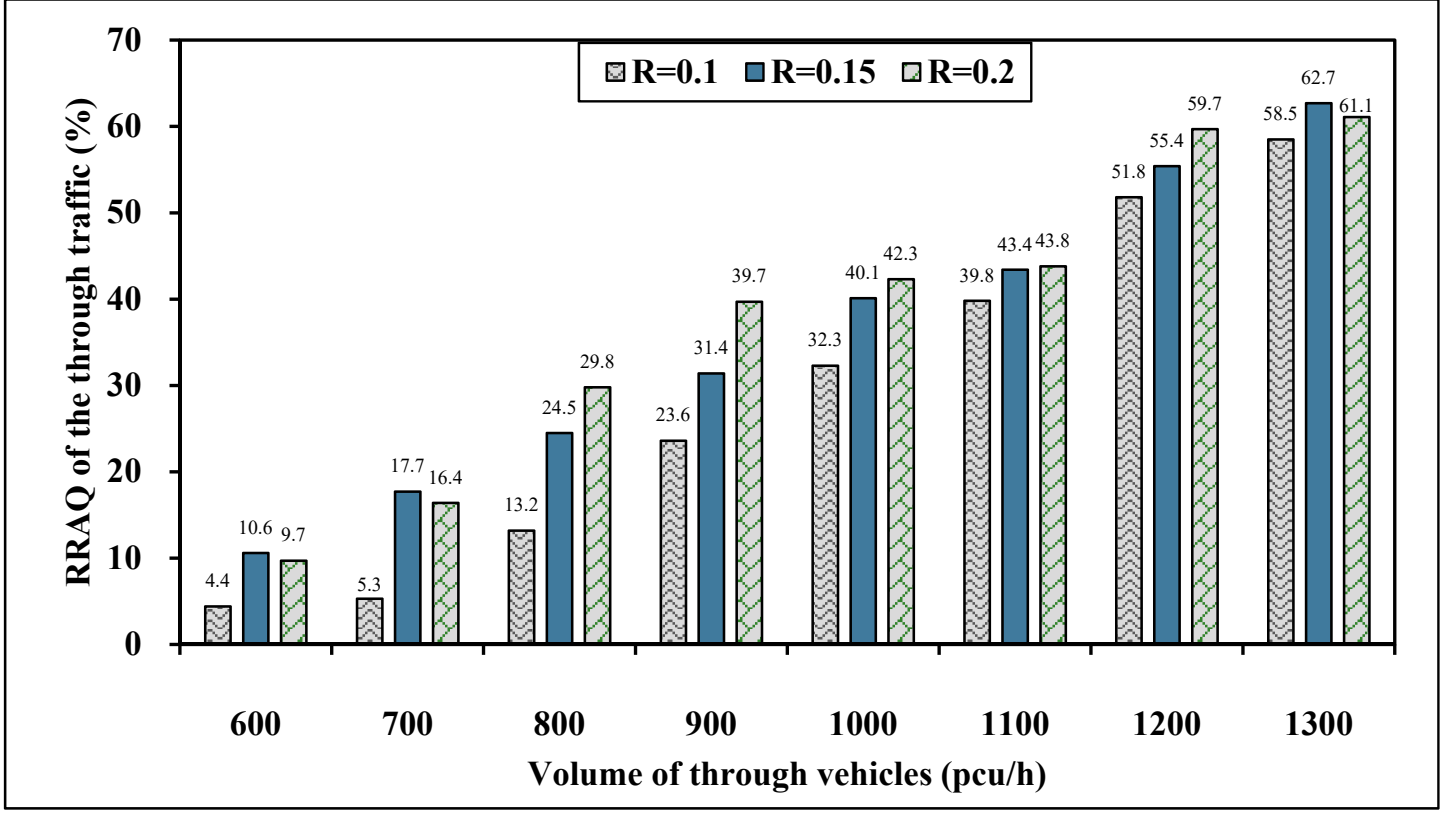

(b)

Figure 11. Impact on queue length: (a) RRAQ of the entire intersection; (b) RRAQ of the through traffic.

Figure 10a,b illustrate the performance of the dynamic lane considering the RRAD of the entire intersection and through vehicles. In Figure 10a, when the ratio of left-turn traffic is fixed, the influence of the dynamic lane on the RRAD of the entire intersection increases with the volume of through traffic. Under a low through volume, especially when the value is more than $900 \mathrm{pcu} / \mathrm{h}$, the value of the RRAD is greater than $10 \%$, which means that the performance of the dynamic lane is sufficient. The maximum RRAD is $34.7 \%$. When the volume is fixed, the influence of the dynamic lane on the RRAD of the entire intersection increases with an increase in the left-turn ratio in most situations. Figure $10 \mathrm{~b}$ reflects the tendency of the RRAD of through vehicles, which is consistent with the RRAD of the entire intersection. Thus, the dynamic lane mainly influences the through vehicles as in the analysis shown in Figure $7 \mathrm{~b}$.

Although the overall trend of Figure 10 is consistent with Figure 7, the RRAD value under the same situation in Figure 10 is smaller than that in Figure 7, and the maximum value is also smaller 
than that of Figure 7. However, in Figure 10, the through flow is larger than that in Figure 7 when the RRAD becomes significant. The reason for this phenomenon is that the merging conflict point was not eliminated in Figure 9. In this case, the dynamic lane acts as a reservoir. When the through volume is small, the effect of dynamic lanes is not significant. When the through volume is large, the queues caused by the merging conflict are stored in the dynamic lane. Therefore, the delay will be effectively reduced compared to that in the intersections without dynamic lanes. However, compared to the situation where the conflict point is eliminated, the merging process will reduce the vehicle speed and increase the delay. This will cause the effect of the dynamic lanes to be discounted and increase the through volume where the dynamic lane becomes significant.

Figure 11a,b illustrate the performance of the dynamic lane considering the RRAQ of the entire intersection and the through vehicles. In Figure 11a, when the ratio of left-turn vehicles is fixed, the influence of the dynamic lane on the RRAQ of the entire intersection increases with the volume of through traffic. Under a low through volume, especially when the value is greater than $900 \mathrm{pcu} / \mathrm{h}$, the value of the RRAQ is greater than $10 \%$, which means that the performance of the dynamic lane is significant. The maximum RRAQ is $47.8 \%$. When the volume is fixed, the influence of the dynamic lane on the RRAQ of the entire intersection increases with an increase in the left-turn ratio in most situations. Figure $11 \mathrm{~b}$ reflects the tendency of the RRAQ for through vehicles, which is consistent with the RRAQ of the entire intersection. Thus, the dynamic lane mainly influences the through vehicles like in the analysis shown in Figure 8b. Similarly, compared to Figure 8, the value of Figure 11 is smaller. This difference can be explained using the same phenomenon of the RRAQ.

\subsection{Analysis of the Dynamic Lane Applicability}

\subsubsection{Analysis of the Critical Volume of Through Vehicles Passing through the Bottleneck}

As shown in Figure 9, at the end of the dynamic lane, the three exit lanes become two lanes, and the traffic flow on the dynamic lane needs to merge into the other two lanes. Therefore, this area becomes a bottleneck. When the through volume is large, queuing may occur due to the existence of a merging conflict. Especially when the traffic load of the other two exit lanes is heavy, it will be difficult for the traffic flow in the dynamic lane to merge normally. If the queue length is too long, it will affect the normal operation of the intersection. Therefore, it is necessary to discuss the critical volume of the through vehicles passing through the bottleneck.

Traffic flow wave theory is an important method to analyze the traffic flow at the bottleneck. Similarly, it is also used to analyze the start and stop of the traffic flow at intersections [51]. In this article, if we take the bottleneck as the research object, the two exit lanes and one dynamic lane can be used as the upstream of the bottleneck, as shown in area $A$ in Figure 12, and two downstream lanes can be used as the downstream area of the bottleneck, as shown in area $B$ in Figure 12. In Figure 12, $Q_{1}$ and $Q_{2}$ are the volumes of $A$ and $B$, respectively; $K_{1}$ and $K_{2}$ are the densities of $A$ and $B$, respectively; $V_{1}$ and $V_{2}$ are the average speeds of $A$ and $B$, respectively; and $V_{W}$ represents the speed of the wave. Moreover, we use $C$ as the capacity of each downstream lane and consider that the vehicles will make full use of the capacity of the downstream lane to drive away from the merging section. Thus, $Q_{2}=2 C$. According to the traffic flow wave theory, $V_{W}$ can be calculated as

$$
V_{W}=\frac{Q_{2}-Q_{1}}{K_{2}-K_{1}}
$$




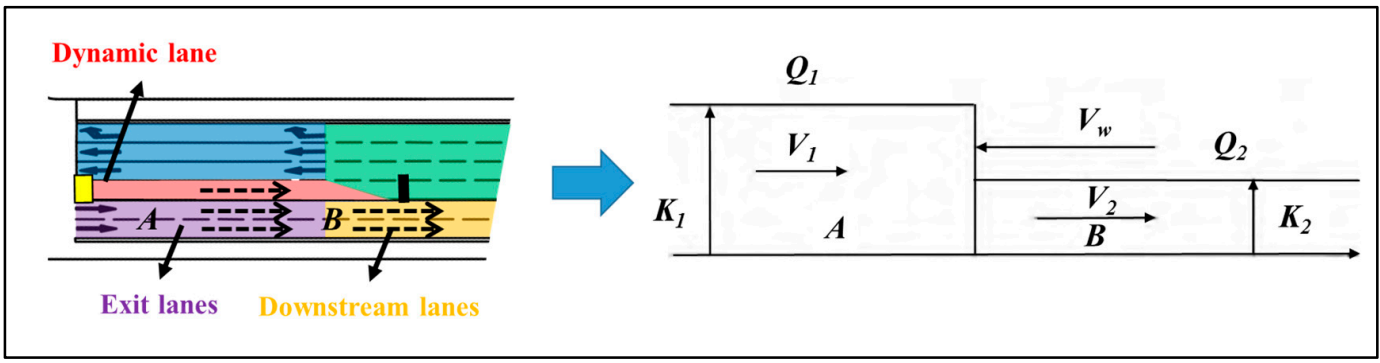

Figure 12. The bottleneck section.

To explore the critical volume of the through vehicles passing through the bottleneck, we must determine under what volume $\left(Q_{1}\right)$ a queue caused by merging will not occur when a dynamic lane is used. As Formula (19) shows, if $V_{W} \leq 0$, the traffic wave will spread to the upstream. If $V_{W} \geq 0$, the traffic wave will stay at the bottleneck or spread to the downstream; under this case, the queue will not occur. Hence, $Q_{1}$ can be obtained as follows:

$$
V_{W} \geq 0 \Rightarrow\left\{\begin{array} { l } 
{ Q _ { 2 } - Q _ { 1 } \geq 0 } \\
{ K _ { 2 } - K _ { 1 } > 0 }
\end{array} \text { or } \left\{\begin{array}{l}
Q_{2}-Q_{1} \leq 0 \\
K_{2}-K_{1}<0
\end{array} .\right.\right.
$$

The first inequality systems describe the movement of one kind of traffic flow that has low volume, low density, and high-speed drivers into another kind of traffic flow that has high volume, high density, and low-speed drivers. However, the second inequality systems describe the movement of a kind of traffic flow with high volume, high density, and low-speed drivers into another kind of traffic flow with low volume, low density, and high-speed drivers. Apparently, the first inequality system is suitable for the merging section we discussed previously. The first inequality system is used as follows:

$$
\left\{\begin{array} { l } 
{ Q _ { 2 } - Q _ { 1 } \geq 0 } \\
{ K _ { 2 } - K _ { 1 } > 0 }
\end{array} \Rightarrow \left\{\begin{array} { c } 
{ 2 C - Q _ { 1 } \geq 0 } \\
{ \frac { 2 C } { V _ { 2 } } - \frac { Q _ { 1 } } { V _ { 1 } } > 0 }
\end{array} \Rightarrow \left\{\begin{array}{c}
Q_{1} \leq 2 C \\
Q_{1}<\frac{2 C V_{1}}{V_{2}}
\end{array}\right.\right.\right.
$$

Generally, when vehicles merge with other vehicles, they will slow down to remain safe, so we can suppose that $V_{1}>V_{2}$. Hence, if $Q_{1} \in[0,2 C]$, a queue caused by merging will not occur.

If $Q_{1}>2 C$, there will be a queue caused by merging. To avoid affecting the normal operations of the intersection, two restricted conditions need to be considered. (1) First, the length of the queue caused by merging should be less than the length of the dynamic lane. (2) The queue must be dissipated before the pre-signal turns green. With two restricted conditions, the range of $Q_{1}$ can be calculated as follows.

(1) First, the length of the queue caused by merging is less than the length of the dynamic lane.

Here, $t$ represents the queue formation time, and $t$ equals the time during which the through vehicles are allowed to drive into the intersection, so $t=g_{2}-l_{1}$ (where $g_{2}$ represents the green time of Phase 2, shown in Figure 4, and $l_{1}$ represents the lost start-up time), $L$ represents the length of the dynamic lane, and $L_{Q}$ represents the average queue length caused by merging. Hence, the relationship between $L_{Q}$ and $L$ can be described as

$$
L_{Q}=\frac{0 \times t+V_{W} \times t}{2} \leq L
$$


so

$$
\left\{\begin{array}{l}
L_{Q}=\frac{0 \times t+\left|V_{W}\right| \times t}{2} \leq L \\
V_{W}=\frac{Q_{2}-Q_{1}}{K_{2}-K_{1}} \\
K_{1}=\frac{Q_{1}}{V_{1}} \\
K_{2}=\frac{Q_{2}}{V_{2}}=\frac{2 C}{V_{2}}
\end{array} \quad \Rightarrow Q_{1} \leq \frac{2 C+\frac{4 C L}{V_{2} t}}{1+\frac{2 L}{V_{1} t}} \Rightarrow Q_{1} \leq \frac{1+\frac{2 L}{V_{2} t}}{1+\frac{2 L}{V_{1} t}} \cdot 2 C,\right.
$$

since $V_{1}>V_{2}, Q_{1}>2 C$. Hence, with the limitation of the queue length, the range of $Q_{1}$ is $Q_{1} \in\left(2 C, \frac{1+\frac{2 L}{V_{2} t}}{1+\frac{2 L}{V_{1} t}} \cdot 2 C\right]$.

(2) The queue must be dissipated before the pre-signal turns green.

According to the signal phase shown in Figure 4, during Phase 3, vehicles from east to north will use the same exit lanes to drive away. During Phase 4, no vehicles will use those exit lanes. $Q_{3}$ represents the volume of this flow. In the worst case, if there is a queue caused by merging and $Q_{3}=Q_{2}$, the queue will not be dissipated until Phase 3 ends. In this case, the time for the queue to dissipate extends only from the beginning of Phase 4 to the beginning of the pre-signal, and we can use $T$ to represent this time. Thus, in the worst case, the queue dissipated time $t^{\prime}$ can be calculated as $t^{\prime}=\frac{\left(Q_{1}-Q_{2}\right) t}{\left|0-Q_{2}\right|}$, where $\left(Q_{1}-Q_{2}\right) t$ is the number of vehicles in the queue, and $\left|0-Q_{2}\right|$ is the dissipated rate of the queue. Thus, the way in which the queue is dissipated before the pre-signal turns green can be described as

$$
\left\{\begin{array}{l}
t^{\prime}=\frac{\left(Q_{1}-Q_{2}\right) t}{\left|0-Q_{2}\right|}<T \\
Q_{2}=2 C
\end{array} \Rightarrow Q_{1}<2 C+\frac{2 C T}{t}\right.
$$

with the restriction of $Q_{1} \in\left(2 C, 2 C+\frac{2 C T}{t}\right)$.

In conclusion, with the two restricted conditions above, the critical volume of through vehicles passing through the bottleneck is

$$
\min \left\{\frac{1+\frac{2 L}{V_{2} t}}{1+\frac{2 L}{V_{1} t}} \cdot 2 C, 2 C+\frac{2 C T}{t}\right\}
$$

where $L$ is the length of the dynamic lane, $V_{1}$ is the average speed of the upstream (Zone A in Figure 12), $V_{2}$ is the average speed of the downstream (Zone B in Figure 12), $t$ is the queue formation time, $C$ is the capacity of each downstream lane, and $T$ is the time for the queue to dissipate. The values of $V_{1}, V_{2}$, and $C$ can be collected from the field. The values of $t$ and $T$ can be obtained from the signal timing case.

If the volume is larger than the critical volume, the dynamic lane would be invalid. However, some measures can be taken to improve the critical volume, such as improving the capacity of the downstream lane, using speed limitations, or adjusting the length of the dynamic lane. If all measures make no sense, a dynamic lane is not suggested to be used at such intersections.

\subsubsection{Analysis of the Potential Risk}

For this type of channelized intersection, the performance of the dynamic lane is illustrated by the simulations. Moreover, an applicability analysis needs to be discussed to enhance the practicality of the dynamic lane. Figure 13 shows two application scenarios of the dynamic lane. Figure $13 \mathrm{a}$ is the intersection shown in Figure 1, and Figure 13b is the intersection shown in Figure 9. Unlike Figure 13a, there is a potential risk of head-on collisions in Figure 13b. In Figure 13a, the green vehicle and the blue vehicle are driving in different lanes independently. However, in Figure 13b, the green vehicle and the blue vehicle are driving in the same lane before the green vehicle changes from the dynamic lane to the downstream lanes. 
Even though the dynamic lane is more strongly recommended for use in the scenario shown in Figure 13a, when it is used in the scenario in Figure 13b, there are three suggestions to decrease the probability of a head-on collision:

(1) Set a speed limit for the dynamic lane. Since a merging conflict still exists, there is no more space for the vehicles to accelerate to a higher speed before merging. Normally, the lower the speed is, the safer the vehicles will be. Thus, a speed limit is suggested to improve the safety and efficiency of the dynamic lane.

(2) Provide manual guidance in the preliminary stage. Generally speaking, a driver needs time to become familiar with new traffic control measures. The provision of manual guidance for the dynamic lane may shorten the time needed before drivers can use the dynamic lane in a familiar way.

(3) Provide warning and guide signs. When the dynamic lane is used for a long time, most drivers will become familiar with the rules. However, some drivers will still be problematic, such as non-local drivers or new drivers. These kinds of signs aim to help such drivers use the dynamic lane successfully.

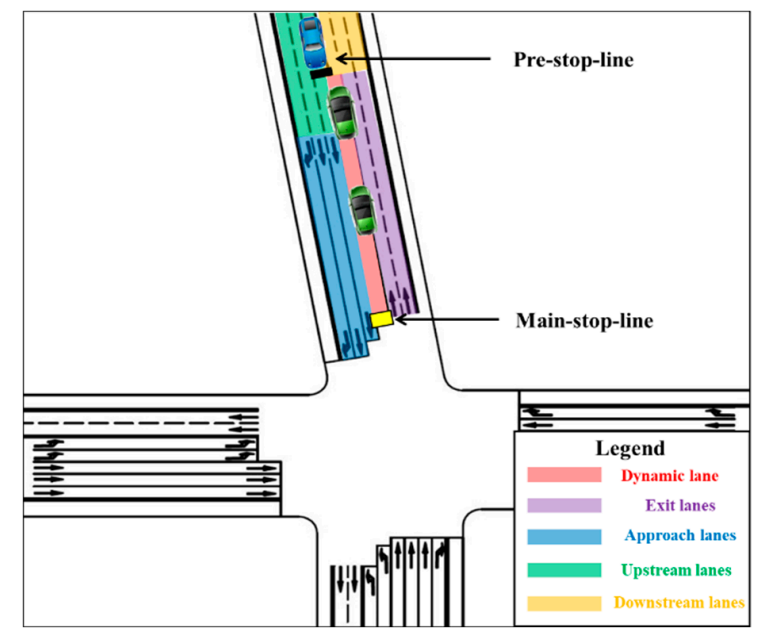

(a)

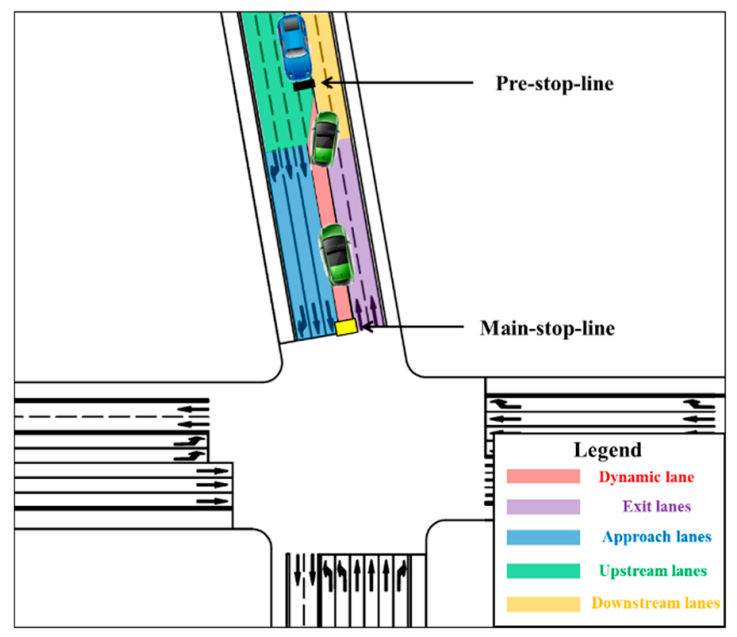

(b)

Figure 13. Two application scenarios of the dynamic lane: (a) intersection shown in Figure 1; (b) intersection shown in Figure 9.

\section{Summary and Conclusions}

This paper proposed a method for the dynamic use of the left-turn lane for opposite through traffic to solve the situation of a quantitative imbalance between the approach and exit lanes. The complete scheme of this method consists of three main parts: the location and routes of the dynamic lane, the temporal scheme, and the spatial scheme. Field data were collected from a typical intersection in the downtown area of Nanjing, P. R. China, for simulation model development. After calibration and validation, the VISSIM models were used to simulate this method for two types of channelized intersections. The results show that the existence of a dynamic lane decreases the average delay and average queue length of the intersection effectively for the intersection with a quantitative imbalance between the approach and exit lanes under the conditions of different through volumes or left-turn ratios. The maximum was $46.9 \%$ (average delay) and $68.3 \%$ (average queue length) for all situations.

Based on the simulation, four major suggestions for the dynamic use of the left-turn lane for opposite through traffic are summarized as follows:

(1) This method is suitable for special intersections where the number of exit lanes is less than that of the approach lanes and where it is difficult to add an auxiliary through lane at the exit due to the limitations of land use. 
(2) An imbalanced intersection should be crossed by two main urban streets with a high traffic volume to ameliorate the limitations of signal control and the length of the dynamic lane. For through volume in the range of $300 \mathrm{pcu} /(\mathrm{h} \bullet l a n e)$ to the upper limit of the capacity, the larger the value is, the more positive effects there are. For the ratio of left-turn traffic, a larger value is preferred along with capacity limits.

(3) Manual guidance is recommended for the preliminary application stage of this method to help drivers become accustomed to the rules of the dynamic lane and decrease the potential safety risks. On the other hand, a speed limit is suggested to be applied for an imbalanced intersection without widening of the left-turn approach lane.

(4) Maintenance should be considered in the decision-making progress. This method needs another traffic signal controller to control the pre-signal. This pre-signal controller must be connected to the main signal controller. Both of them should use the same signal scheme. Moreover, some other signs (like guidance, warning, and limitations) are also needed. These signs will guide drivers to use the dynamic lane successfully and remind drivers of possible merging conflicts and speeding limitations. The costs of the maintenance depends on the reality of the application.

Based on the results of this research, it would be worthwhile to more deeply research the influence of violations on intersections using this method. It is impossible to avoid violations completely, such as skipping red signals and lane switching from other lanes. None of these violations would influence the normal operational order negatively, but it is unknown how negative these influences would ultimately be. If this method is applied in the future, the evaluation of such violations could be researched using field data. In contrast, the simulation method is recommended if it is difficult to collect field data for this method.

Author Contributions: Conceptualization, Y.Z. and W.W.; investigation, Y.Z., X.H., and J.X.; methodology, Y.Z. and X.H.; writing — original draft, Y.Z.; writing - review and editing, J.X. and D.L. All authors have read and agreed to the published version of the manuscript.

Funding: This work was supported by the National Natural Science of China $(51878166,71801042)$ and the Natural Science of Jiangsu Province (BK20180381).

Acknowledgments: The authors are grateful to the editor and anonymous reviewers of this paper and give special thanks to the MDPI professional language editing services for ensuring that the English grammar is free of mistakes in this manuscript.

Conflicts of Interest: The authors declare no conflict of interest.

\section{References}

1. Qi, H.; Wang, D.; Chen, P.; Bie, Y. Simulation of departure flow profile at stop lines for signal approach spillover. Transp. Res. Part C Emerg. Technol. 2013, 33, 88-106. [CrossRef]

2. Wang, Y.; Rong, J.; Zhou, C.; Chang, X.; Liu, S. An Analysis of the Interactions between Adjustment Factors of Saturation Flow Rates at Signalized Intersections. Sustainability 2020, 12, 665. [CrossRef]

3. Hummer, J.E. Unconventional Left-Turn Alternatives for Urban and Suburban Arterials-Part One. ITE J. Inst. Transp. Eng. 1998, 68, 26-29.

4. Hummer, J.E. Unconventional Left Turn Alternatives for Urban and Suburban Arterials. Part Two. ITE J. Web 1998, 68, 101-106.

5. Hummer, J.E.; Reid, J.D. Unconventional Left-Turn Alternatives for Urban and Suburban Arterials: An Update. In Proceedings of the 79th Annual Meeting of the Transportation Research Board, Dallas, TX, USA, 28-30 June 1999.

6. Wilbur Smith Associates. Innovative Intersections: Overview and Implementation Guidelines. Community Planning Association of Southwest Idaho; Wilbur Smith Associates: Meridian, ID, USA, 2008.

7. Hughes, W.; Jagannathan, R.; Sengupta, D.; Hummer, J. Alternative Intersections/Interchanges: Informational Report. FHWA-HRT-09-060. FHWA; U.S. Department of Transportation: Washington, DC, USA, 2010.

8. Al-Salman, H.S.T.; Salter, R.J. The Control of Right Turning Vehicles at Signal Controlled Intersections. Traffic Engineering and Control; Printerhall Limited: London, UK, 1974; Volume 15, pp. 683-686. 
9. Goldblatt, R.; Mier, F.; Friedman, J. Continuous Flow Intersections. ITE J. 1994, 64, 35-42.

10. Xuan, Y.; Daganzo, C.F.; Cassidy, M.J. Increasing the capacity of signalized intersections with separate left turn phases. Transp. Res. Part B Methodol. 2011, 45, 769-781. [CrossRef]

11. Xuan, Y.; Gayah, V.; Daganzo, C.; Cassidy, M. Multimodal Traffic at Isolated Signalized Intersections: New Management Strategies to Increase Capacity. UC Berkeley: Center for Future Urban Transport: A Volvo Center of Excellence. 2009. Available online: https://escholarship.org/uc/item/7fk7j154 (accessed on 26 August 2020).

12. Tabernero, V.; Sayed, T. Upstream Signalized Crossover Intersection: An Unconventional Intersection Scheme. J. Transp. Eng. 2006, 132, 907-911. [CrossRef]

13. Reid, J.D.; Hummer, J.E. Travel Time Comparisons between Seven Unconventional Arterial Intersection Designs. Transp. Res. Rec. J. Transp. Res. Board 2001, 1751, 56-66. [CrossRef]

14. Lo Faro, G.; Tripodi, A. The Intersection Problem for Twin Bowtie and Near Bowtie Systems. ARS Comb. 2001, 61, 245-254.

15. Haley, R.L.; Ott, S.E.; Hummer, J.E.; Foyle, R.S.; Cunningham, C.M.; Schroeder, B.J. Operational Effects of Signalized Superstreets in North Carolina. Transp. Res. Rec. J. Transp. Res. Board 2011, 2223, 72-79. [CrossRef]

16. Naghawi, H.H.; Idewu, W.I.A. Analyzing Delay and Queue Length Using Microscopic Simulation for the Unconventional Intersection Design Superstreet. J. South Afr. Inst. Civ. Eng. 2014, 56, 100-107.

17. Ott, S.E.; Haley, R.L.; Hummer, J.E.; Foyle, R.S.; Cunningham, C.M. Safety effects of unsignalized superstreets in North Carolina. Accid. Anal. Prev. 2012, 45, 572-579. [CrossRef] [PubMed]

18. Yan, Y.; Qu, X.; Li, H. On the design and operational performance of waiting areas in at-grade signalized intersections: An overview. Transp. A Transp. Sci. 2018, 14, 901-928. [CrossRef]

19. Guell, D.L. Additional Through Lanes at Signalized Intersections. J. Transp. Eng. 1983, 109, 499-505. [CrossRef]

20. Tarawneh, S.M.; Tarawneh, T.M. Effect on utilization of auxiliary through lanes of downstream right-turn volume. J. Transp. Eng.-ASCE. 2002, 128, 458-464. [CrossRef]

21. Lee, J.; Hummer, J.E.; Rouphail, N. False Capacity for Lane Drops (Final report); FHWA/NC/2005-01; North Carolina Department of Transportation: Raleigh, NC, USA, 2005.

22. Zhao, J.; Ma, W.; Zhang, H.M.; Yang, X. Increasing the Capacity of Signalized Intersections with Dynamic Use of Exit Lanes for Left-Turn Traffic. Transp. Res. Rec. J. Transp. Res. Board 2013, 2355, 49-59. [CrossRef]

23. Zhao, J.; Liu, Y.; Di, D. Optimization model for layout and signal design of full continuous flow intersections. Transp. Lett. 2016, 8, 194-204. [CrossRef]

24. Liu, Z.-H.; Wan, J.; Wang, W.; Li, Z. Evaluating the Impacts of Unconventional outside Left-Turn Lane Design on Traffic Operations at Signalized Intersections. Transp. Res. Rec. J. Transp. Res. Board 2011, 2257, 62-70. [CrossRef]

25. Zhao, J.; Yu, J.; Zhou, X. Saturation Flow Models of Exit Lanes for Left-Turn Intersections. J. Transp. Eng. Part. A: Syst. 2019, 145, 04018090. [CrossRef]

26. Zhao, Y.; James, R.M.; Xiao, L.; Bared, J. A Capacity Estimation Model for a Contraflow Left-Turn Pocket Lane at Signalized Intersections. Transp. Res. Rec. J. Transp. Res. Board 2018, 2672, 22-34. [CrossRef]

27. Su, P.; Krause, C.; Hale, D.; Bared, J.; Huang, Z.T. Operational Advantages of Contraflow Left-turn Pockets at Signalized Intersections. ITE J. Inst. Transp. Eng. 2016, 86, 44-49.

28. Chen, S.; Li, X.S.; Wang, Y.H.; Ren, Y.Y. Traffic Control Plan Optimization for the Intersection with Contraflow Left-Turn Lane. J. Harbin Inst. Technol. 2018, 50, 74-82.

29. Chen, Q.; Yi, J.X.; Wu, Y.L. Cellular Automaton Simulation of Vehicles in the Contraflow Left-Turn Lane at Signalized Intersections. IET Intell. Transp. Syst. 2019, 13, 1164-1172. [CrossRef]

30. Liu, P.; Wu, J.; Zhou, H.; Bao, J.; Yang, Z. Estimating Queue Length for Contraflow Left-Turn Lane Design at Signalized Intersections. J. Transp. Eng. Part. A Syst. 2019, 145, 04019020. [CrossRef]

31. Wu, J.; Liu, Z.-H.; Tian, Z.; Xu, C. Operational analysis of the contraflow left-turn lane design at signalized intersections in China. Transp. Res. Part C Emerg. Technol. 2016, 69, 228-241. [CrossRef]

32. Wu, J.; Liu, Z.-H.; Qin, X.; Zhou, H.; Yang, Z. Developing an actuated signal control strategy to improve the operations of contraflow left-turn lane design at signalized intersections. Transp. Res. Part C Emerg. Technol. 2019, 104, 53-65. [CrossRef]

33. Zhao, J.; Liu, Y. Safety evaluation of intersections with dynamic use of exit-lanes for left-turn using field data. Accid. Anal. Prev. 2017, 102, 31-40. [CrossRef] 
34. Zhao, J.; Yun, M.; Zhang, H.M.; Yang, X. Driving simulator evaluation of drivers' response to intersections with dynamic use of exit-lanes for left-turn. Accid. Anal. Prev. 2015, 81, 107-119. [CrossRef]

35. Guo, Y.; Sayed, T.; Zaki, M.H.; Liu, Z.-H. Safety evaluation of unconventional outside left-turn lane using automated traffic conflict techniques. Can. J. Civ. Eng. 2016, 43, 631-642. [CrossRef]

36. Transportation Research Board of the National Academies. Chapter 18 Signalized Intersections, Highway Capacity Manual 2010; Transportation Research Board: Washington, DC, USA, 2010; Volume 3, pp. $18-31$.

37. Daniel, L.; Matthew, J.H. Transportation Research Board, National Council. In Chapter 2 Statistical Distribution of Traffic Characteristics. Traffic Flow Theory: A Monograph Special Report; Transportation Research Board, National Council: Washington, DC, USA, 2010; p. 17.

38. AutoNavi Traffic Big-Data. 2020. Traffic Analysis Reports for Major Cities in China (2020 Q2). Available online: https://report.amap.com/share.do?id=8b8f04f7731fb5e40173702622ea20fe (accessed on 26 August 2020).

39. Dadashzadeh, N.; Ergun, M.; Kesten, S.; Žura, M. An Automatic Calibration Procedure of Driving Behaviour Parameters in the Presence of High Bus Volume. Promet. TrafficTransp. 2019, 31, 491-502. [CrossRef]

40. Dadashzadeh, N.; Ergun, M. An Integrated Variable Speed Limit and ALINEA Ramp Metering Model in the Presence of High Bus Volume. Sustainability 2019, 11, 6326. [CrossRef]

41. Wang, C.; Xu, C.; Xia, J.; Qian, Z.; Lu, L. A combined use of microscopic traffic simulation and extreme value methods for traffic safety evaluation. Transp. Res. Part C Emerg. Technol. 2018, 90, 281-291. [CrossRef]

42. Huang, F.; Liu, Z.-H.; Yu, H.; Wang, W. Identifying if VISSIM simulation model and SSAM provide reasonable estimates for field measured traffic conflicts at signalized intersections. Accid. Anal. Prev. 2013, 50, 1014-1024. [CrossRef]

43. Mathew, T.V.; Radhakrishnan, P. Calibration of Microsimulation Models for Nonlane-Based Heterogeneous Traffic at Signalized Intersections. J. Urban. Plan. Dev. 2010, 136, 59-66. [CrossRef]

44. Hua, X.; Wang, Y.W.; Zhu, W.; Wang, Y.; Wang, W.; Hua, Y.Z. Control Strategy Optimization for Two-Lane Highway Lane-Closure Work Zones. Sustainability 2019, 11, 4567. [CrossRef]

45. Yang, M.; Ding, J.; Wang, W.; Ma, Y.-Y. A coordinated signal priority strategy for modern trams on arterial streets by predicting the tram dwell time. KSCE J. Civ. Eng. 2017, 22, 823-836. [CrossRef]

46. Mohan, M.; Chandra, S. Queue clearance rate method for estimating passenger car equivalents at signalized intersections. J. Traffic Transp. Eng. 2017, 4, 487-495. [CrossRef]

47. Liu, P.; Qu, X.; Wang, W.; Cao, B. Using VISSIM to Model Capacity of U-turns at Unsignalized Intersections with Non-traversable Median Cross Sections. In Proceedings of the 89th Annual Meeting of the Transportation Research Board, Washington, DC, USA, 10-14 January 2010.

48. Saha, A.; Chandra, S.; Ghosh, I. Modeling Platoon Dispersion at Signalized Intersections in Mixed Traffic Scenario. Arab. J. Sci. Eng. 2018, 44, 4829-4838. [CrossRef]

49. Zhang, H.; Liu, H.X.; Chen, P.; Yu, G.; Wang, Y. Cycle-Based End of Queue Estimation at Signalized Intersections Using Low-Penetration-Rate Vehicle Trajectories. IEEE Trans. Intell. Transp. Syst. 2019, 21, 3257-3272. [CrossRef]

50. Liu, S.; Li, Y.; Qiu, Y.; Zhang, B.; Qiu, S.; Liu, X. Signal Timing Optimization Algorithm for an Intersection Connected with an Urban Expressway. Arab. J. Sci. Eng. 2020, 1-18. [CrossRef]

51. Daniel, L.; Matthew, J.H. Transportation Research Board, National Council. In Chapter 7 Hyderodynamic and Kinematic Models of Traffic. Traffic Flow Theory: A Monograph Special Report; Transportation Research Board, National Council: Washington, DC, USA, 2010; pp. 112-117.

(C) 2020 by the authors. Licensee MDPI, Basel, Switzerland. This article is an open access article distributed under the terms and conditions of the Creative Commons Attribution (CC BY) license (http://creativecommons.org/licenses/by/4.0/). 